\title{
Alpha-Particle Effects on High-n Instabilities in Tokamaks
}

G. Rewoldt

Plasma Physics Laboratory, Princeton University, Princeton, New Jersey 08513

\begin{abstract}
Hot $\alpha$-particles and thermalized helium ash particles in tokamaks can have significant effects on high toroidal mode number instabilities such as the trappedelectron drift mode and the kinetically calculated magnetohydrodynamic ballooning mode. In particular, the effects can be stabilizing, destabilizing, or negligible, depending on the parameters involved. In high-temperature tokamaks capable of producing significant numbers of hot $\alpha$-particles, the predominant interaction of the mode with the $\alpha$-particles is through resonances of various sorts. In turn, the modes can cause significant anomalous transport of the $\alpha$-particles and the helium ash. Here, sesults of comprehensive linear eigenfrequency-eigenfunction calculations are presented for relevant realistic cases to show these effects.
\end{abstract}

\section{DISCLAIMER}

This report was prepared as an account of work sponsored by an agency of the United States This report was prepared as an account of werk spornment nor ony asency thereof, nor any of their employees, makes any warranty, express or impliod, or assumes any legal liability or responsiemployees, makes any warranty, express or impliod, or assumes asy legal liablity product, or bility for the aceracy, conpels that its use would not infringe privately owned rights. Referprocess disclosed, or represents that its use woyld not infinge prise frode name, trademark, ence herein to any specifise does not necessarily constitute or imply its endorsement, recommanufacturer, or otherwise does United States Government or any agency thereof. The views mendation, or fasoring by the Unith herein do not necessarily state or reflect those of the and opinions of authors expressed herein do notes Goverpment or any agency thereof. 


\section{INTRODUCTION}

Application of a comprehensive kinetic linear eigenfrequency ejgenfunction calculation ${ }^{1}$ fur high-n (toroidal mode number) instabilities in tokamaks to look at the effects of hot $\alpha$-particles $(\alpha$ 's) and thermalized helium ash particles $(\mathrm{He})$ on preexisting instabilities yields a number of interesting results. These are the trapped-electron drift mode in the drift branch and the kinetically calculated magnetohydrodynamic (MHD) ballooning mode in the shear-Alfven branch. Application is made to two tokamaks which have the potential for producing significant populations of $\alpha$-particles, namely the proposed Compact Ignition Tokamak (CIT) and the Tokamak Fusion Test Reactor ${ }^{3}$ (TFTR). The linear and quasilinear calculation is fully electromagnetic and includes all of the relevant kinetic effects for the magnetically trapped and untrapped (circulating, passing) particles tor each species, including bounce-frequency resonances for the trapped particles, transit-frequency resonances (Landau damping) for the untrapped particles, magnetic (gradient and curvature) drift frequency resonances, and full finite Larnor radius effects. None of these effects, except for the trapped- $\alpha$ time-average magnetic drift frequency resonances, are included in previous calculations ${ }^{4-9}$ of $\alpha$-particle effects on high-n instabilities, so the present calculation represents an extension. There are also related calculations concentrating on the effects of hot beam ions, rather than $\alpha$-particles, without ${ }^{10}$ and with ${ }^{11}$ transit-frequency resonances fo: untrapped bearn ions. The present calculation employs the so-called ballooning formalism ${ }^{1}$ for high- $n$ toroidal modes, to lowest order in $1 / n$, and accordingly the calculation is local to a chosen magnetic surface and involves a system of three one-dimensional integrodifferential equations along the unperturbed magnetic field lines.

For the two modes considered, which can be unstable ever in the absence of $\alpha$-particles, the effects of the $a$-particles and the helium ash particles can be stabilizing, destabilizing, or negligible, depending on the parameters involved. For the CIT case considered here, the trapped-electron drift mode interacts only weakly with the $\alpha$-particles and the helium ash

- "particles. For the same case, the kinetically calculated MHD ballooning mode is strongly 
destabilized by the a-particles, which lower the critical value of $\beta \equiv$ (plasma pressure / magnetic pressure) for instability substantially, but is only weakly affected by the helium ash particles. Both modes cause significant quasilinear transport of the helium ash particles and energy, but only the MHD ballooning mode causes significant quasilinear transport of the hot- $\alpha$ particles and energy. For the TFTR case, the effects of the $\alpha$-particles and helium on the two modes are similar, except that the critical $\beta$ value for the WHD ballooning mode is not lowered significantly, and the quasilinear transport of the $\alpha$-particles and helium is negligible.

The eigenmode equations solved and the solution methods are described in detail in Ref. 1, so this information will not be repeated here. The input data for the calculation come from results of runs of the BALDUR tokamak transport code, ${ }^{12}$ which provides radial profiles of particle number density $n_{j}(r)$ and temperature $T_{j}(r)$ for each species labeled by $j$ and also of the safety factor $g(r)$. In general, up to four particle species are included: $j=e$ for background electrons, $j=i$ for background hydrogenic ions of mass $m_{i}=2.5$ amu, representing an equal mixture of deuterium and tritium, $j=\mathrm{He}$ for the thermalized helium ash, and $j=\alpha$ for the hot $\alpha$-particles which are still in the process of slowing down from their birth energy, $E_{\alpha}=3.52 \mathrm{MeV}$, to the backgtound ion temperature. Of course, $j=\alpha$ and $j=$ He refer to the same kind of particles, but it clarifies their effects and is numerically convenient to treat them ss two separate populations. The equilibrium distribution function $F_{j}$ for each species except the $\alpha$ 's is taken to be Maxwellian,

$$
F_{j}=F_{m j} \equiv \frac{n_{j}(r)}{\left[2 \pi T_{j}(r) / m_{j}\right]^{3 / 2}} \exp \left[-E / T_{j}(r)\right]
$$

which is a very good approximation. For the $\alpha$-particles, two different equilibrium distribution functions are employed, $F_{\alpha}=F_{m \alpha}$ and

$$
F_{\alpha}=F_{S D} \equiv \frac{n_{\alpha}(r)}{v^{3}+v_{c}^{3}} \frac{3 / 4 \pi}{\ln \left(1+v_{\alpha}^{3} / v_{c}^{3}\right)} H\left(v_{\alpha}-v\right)
$$


the so-called slowing-down distribution, ${ }^{13}$ where $v$ is the magnitude of the particle velocity, $v_{\alpha} \equiv\left(2 E_{\alpha} / m_{\alpha}\right)^{1 / 2}, v_{e} \equiv\left(2 T_{e} / m_{e}\right)^{1 / 2}, v_{e} \equiv\left(3 \pi^{1 / 2} m_{e} Z_{1} / 4 m_{\alpha}\right)^{1 / 3} v_{e}$ is the so-called critical velocity, $Z_{1} \equiv \sum_{j \neq e}\left(n_{j} m_{\alpha} Z_{j}^{2} / n_{e} m_{j}\right), Z_{j}$ is the charge number, and $H(x)$ is the usual Heaviside step function. The actual $\alpha$-particle equilibrium distribution is probably close to $F_{S D}$, but comparing results for $F_{\alpha}=F_{S D}$ and $F_{\alpha}=F_{m \alpha}$ gives an estimate of the sensitivity of the results to this choice. The MHD equilibrium is then calculated numerically using the PEST equilibrium code ${ }^{4}$ with pressure and $q$ profiles obtained from the BALDUR results at a chosen time in the discharge. This approach is useful, in particular, for the CIT device, which has a strongly shaped cross section, with a vertical elongation factor of about two and significant triangularity.

The previous work most relevant to the present calculation is that of Spong et al., in Refs. 5 to 9 . In Ref. 5 , the equation employed is the usual $\boldsymbol{n} \rightarrow \infty$ ideal-MHD ballooning mode equation including the diamagnetic drift frequency $\omega_{\star p i}$, generalized to allow for a hot species that is anisotropic in pitch angle, and including an additional term corresponding to the trapped-a-particle time-average magnetic drift frequency resonance term. It is assumed in this reference that all of the $\alpha$-particles are deeply trapped in pitch angle, so that there are no untrapped $\alpha$ 's, and that the $\alpha$ 's are Maxwellian in energy. The so-called "s- $\alpha$ " mode] MHD equilibrium ${ }^{15}$ is employed. In Ref. 6, the equation is modified to replace the Maxwellian energy distribution by the slowing-down energy distribution. In Ref. 7, the trapped- $\alpha$ distribution is changed from all deeply trapped to uniform in pitch-angle, but still without untrapped $\alpha$ 's. In Refs. 8 and 9, a more general model MHD equilibrium is employed which allows for vertical elongation and triangularity. The conclusion in these papers is that the $\alpha$-particles can be significantly stabilizing or destabilizing for the ideal MHD ballooning mode, depending on the parameters considered.

In the present calculation, there are a number of significant extensions to the physics contained in Refs. 5 to 9 . Results are given for the trapped-electron drift mode, which is expected to be dominant at lower values of $\beta$, as well as for the kinetically calculated 
MHD ballooning mode, which may be dominant at higher values of $\beta$. Relative values of the quasilinear particle and energy diffusion coefficients for the different species are given, along with the linear growth rates and real frequencies. Untrapped $\alpha$-particles as well as trapped $\alpha$ particles are retained (as with the other species), along with the transit-frequency resonances (Landau damping) for untrapped particles and the bounce-frequency resonances for trapped particles. This also.allows the possibility of "hybrid" resonances, where both the magnetic drift frequency and some multiple of the bounce or transit frequency are involved. Full finite Larmor radius effects are retained. Thermalized helium ash as well as the hot $\alpha$-particles are included. Quantitative results showing the importance of some of these additional physical effects will be given in the next section.

In Sec. II, parameters and results are given in detail for a CIT case. In Sec. III, they are given more briefly for a TFTR case. Conclusions and discussion are given in Sec. IV.

\section{CIT}

The design for the proposed CIT device has been modified several times. The revised design $^{2}$ considered here has (vacuum) major radius $R_{0}=1.75 \mathrm{~m}$, toroidal magnetic field $B_{0}=10 \mathrm{~T}$, and plasma current $I_{p}=9 \mathrm{MA}$. For this revised design, trapped- $\alpha$-particle orbit losses due to magnetic ripple are negligible, ${ }^{16}$, so the $\alpha$-particle distribution in pitch angle is isotropic to a good approximation, and both trapped and untrapped $\alpha$-particles will be present. In a BALDUR transport code $\operatorname{cun}^{17}$ for this revised design, a time in the flat top period for plasma quantities is chosen that is between sawtooth crashes. The magnetic surfaces for the MHD equilibrium are shown in Fig. 1. A magnetic surface with (average) minor radius $r \simeq a / 2$ is chosen, which is somewhat outside the $q=1$ surface, where $a$ is the plasma edge radius. On this surface, the local parameters are: $\beta=\beta_{\mathrm{CIT}} \equiv \beta_{\mathrm{c}}+\beta_{i}+$ $\beta_{i k}=5.82 \%, \beta_{j} \equiv 8 \pi n_{j} T_{j} / B_{0}^{2}, q=1.17, \hat{s} \equiv r(d q / d r) / q=0.456, \epsilon_{0} \equiv r / R_{0}=0.154$, $R_{0}=180 \mathrm{~cm}, r_{n e} / r=1.62, r_{n j} \equiv-\left(d \ln n_{j} / d r\right)^{-1}, \eta_{j} \equiv\left(d \ln T_{j} / d r\right) /\left(d \ln n_{j} / d r\right), \nu_{*}^{E} \equiv$ effective electron collision frequency/average trapped electron bounce frequency $=0.0035$, 
and $n_{e}=3.71 \times 10^{14} \mathrm{~cm}^{-3}$. Other local parameters for the four species are given in Table I. To put the $a$-particle and helium effects in perspective. note that

$$
\frac{n_{a}}{n_{e}} \simeq 0.5 \%, \frac{\beta_{a}}{\beta_{\mathrm{CIT}}} \simeq 12 \%, \frac{\beta_{a}^{\prime}}{\beta_{\mathrm{CIT}}^{\prime}} \simeq 25 \%
$$

while

$$
\frac{n_{H e}}{n_{e}} \simeq 9 \%, \frac{\beta_{H k}}{\beta_{\mathrm{CII}}} \simeq 4 \%, \frac{\beta_{H k}^{\prime}}{\beta_{\mathrm{CIT}}^{\prime}} \simeq 6 \%,
$$

where $\beta_{j}^{\prime} \equiv d \beta_{j} / d r$ and $\beta_{\mathrm{CrT}}^{\prime} \equiv \beta_{\mathrm{e}}^{\prime}+\beta_{i}^{\prime}+\beta_{H}^{\prime}$. In addition to mode frequencies computed for this standard set of parameters, results will also be presented as the local $\beta$ is varied artificially from $\beta_{\text {cIT }}$. Specifically, for each different $\beta$ value, the entire BALDUR pressure profile is multiplied by $\beta / \beta_{\text {CIT }}$ and the numerical $M H D$ equilibrium is recomputed. Also, $q(\psi)$ is held fixed, so this constitutes a flux-conserving sequence. As $\beta$ is varied, the Shafranov shift and the magnetic curvature change correspondingly. It is this variation which is responsible for the so-called "second stability region" for the MHD ballooning mode. When $\beta$ is varied, the $r_{\beta} \equiv-\left(d \ln \beta / d_{r}\right)^{-1}, T_{j}, n_{j} / \pi_{e}, B_{0}$, and $n$ are normally held. fixed, so that all of the $n_{j}$ vary proportional to $\beta$. However, the $n_{j}$ enter the calculation directly only through the collision frequencies, and collisions are essentially negligible for this case (and are normally not included). Thus, the main effect of the $\beta$ variation is through the MHD equilibrium, as has been described, and indirectly through the Alfvén frequency, $\omega_{A} \equiv v_{A} / q R_{0} \equiv\left[B_{0} /\left(4 \pi \sum_{j} n_{j} m_{j}\right)^{1 / 2}\right] / q R_{0}$. If a different choice had been made, for instance if $B_{0}$ were to vary proportional to $\beta^{-1 / 2}$ with the $n_{j}$ and $T_{j}$ held fixed, the effect of the $\beta$ variation would be somewhat different, but the final results would not differ greatly from those with the present choice, as was seen in Ref. 18. Alternatively, $T_{j}$ could be chosen to vary with $T_{j} \propto \beta$, holding $\tau_{\beta}, n_{j}, B_{0}$, and $n$ fixed. More complicated forms of variation could also be considered. In addition, the toroidal mode number $n \propto k_{\theta} \rho_{j}$, where $k_{\theta}$ is the poloidal wave number and $\rho_{j}$ is the gyroradius, is varied to find values at or near those which maximize the growth rate. Other parameters are varied to explore their effects also. 
Linear frequencies for the trapped-electron drift mode (TEM) and the kinetically calculated MHD ballooning mode (kMHDBM) for the eigenmodes with the fewest (zero) nodes along the magnetic field line are given in Table II in units of $\omega_{. e}$, the electron diamagnetic drift frequency, for several $\beta \propto n_{j}$ and $n$ values. The results show the effects of turning on and off the contributions of the hot $\alpha$-particle and the thermalized helium ash species. In doing this, the requirements of (equilibrium) charge neutrality and of its radial derivatjve must always be satisfied; this is accomplished by the arbitrary choice of adjusting $n_{i} / n_{e}$ and $r_{n i} / r_{n e}$ as each species is turned on or off. Results are given for the TEM and the kMHDBM for $\beta=\beta_{\text {CrT }}=5.80 \%$, which is below the simplest ideal MHD ballooning mode critical $\beta$, $\beta_{c 1}^{\mathrm{MHD}} \simeq 8.25 \%$, the boundary of the so-called first stability region, and for the kMHDBM for $\beta=11.3 \%$, which is above $\beta_{\mathrm{el}}^{\mathrm{MHD}}$.

Comparing the different rows of Table 11, it can be seen that turning on the $\alpha$-particles with $F_{\alpha}=F_{m \alpha}$ and $\eta_{a}=0.118$ has only a slight effect on the TEM, is strongly destabilizing for the kMHDBM for $\beta<\beta_{c 1}^{M H D}$, and is somewhat stabilizing for $\beta>\beta_{c 1}^{M H D}$. Changing from $\eta_{a}=0.118$ to $\eta_{\alpha}=0$ has little effect on the results, implying that the $\alpha$-particle density gradient is dominant over the $\alpha$-particle temperature gradient. Changing from the Maxwellian distribution function $F_{m a}$ with $\eta_{\alpha}=0$ to the slowing-down distribution function $\dot{F}_{S D}$ with $\eta_{\alpha}=0$ for the $\alpha$ 's has little effect on the TEM, increases the $\alpha$-particle destabilization for the kMHDBM somewhat for $\beta<\beta_{\mathrm{c} 1}^{\mathrm{MHD}}$, and destabilizes the KMHDBM for $\beta>\beta_{\mathrm{cl}}^{\text {MHD }}$. Including the helium ash as well as the $\alpha$-particles has very little effect on the TEM, destabilizes the kMHDBM slightly for $\beta<\beta_{\mathrm{cl}}^{\mathrm{MHD}}$, and stabilizes it very slightly for $\beta>\beta_{\mathrm{c1}}^{\mathrm{MHD}}$.

Thus, the most important effect in this case is the strong $\alpha$-particle destabilization of the kMHDBM for $\beta<\beta_{e 1}^{\text {MHD }}$. The causes for this destabilization are explored in more detail in Table III, which shows the change in the growth rate $\gamma$ and the real frequency $\omega_{r}$ coming from each of the $\alpha$-particle terms or effects, for $\beta=\beta_{\mathrm{CIT}}=5.82 \%, n=19, k_{\mathrm{\theta}} \rho_{i}=0.12$, $k_{\theta} \rho_{\alpha}=0.51, F_{\alpha}=F_{S D}$, and $\eta_{\alpha}=0$, with no helium. The top three rows in Table III are 
of most interest. All three of these $\alpha$-particle terms are destabilizing, but the untrapped$\alpha$-particle term is the biggest, being about twice as large as the total (time-average plus non-time-average) trapped- $\alpha$ term. For the trapped- $\alpha$ terms, also, the non-tirne-average term is about twice as large as the time-average term. This trapped- $\alpha$ time-average term is the only one of these three $\alpha$-particle terrns included in Refs. + to 9 . Thus, the results in these references for $\alpha$-particle effects on the MHD ballooning mode would have the right sign, but be considerably too small, for cases like this CIT case.

To understand better physically why such a small fraction of $\alpha$-particles, $n_{\alpha} / n_{e} \simeq 0.5 \%$, can have an order unity effect on the growth rate, it is necessary to understand the nature of the resonances between the mode and the $\alpha$-particles. For the case considered in Table III, the relevant orderings for the $\alpha$-particles are:

$$
\omega_{a \alpha} \gg \bar{\omega}_{l a} \simeq\left|\omega_{r}\right|>\bar{\omega}_{b a}>\bar{\omega}_{d a} \gg \gamma,
$$

$k_{\ominus} \rho_{\alpha}=O(1)$, and $k_{\|} L_{c}=O(1)$, where $\omega_{* \alpha}$ is the $\alpha$-particle diamagnetic drift frequency, $\bar{\omega}_{t o}$ is the average untrapped- $\alpha$-particle transit frequency, $\omega_{T}$ is the mode real frequency, $\bar{\omega}_{b a}$ is the average trapped- $\alpha$-particle bounce frequency, $\bar{\omega}_{d a} \equiv\left(r_{n o} / R_{0}\right) \omega_{* \alpha}$ is a measure of the average trapped- $\alpha$-particle time-average magnetic drift frequency, $\gamma$ is the growth rate, $k_{\|}$is the wave number along the equilibrium magnetic field line, and $L_{c}=O\left(q R_{0}\right)$ is the connection length. The form of the resonant denominator for untrapped particles is ${ }^{1}$

$$
G_{t} \equiv\left(\omega+i \nu_{f}-\kappa \omega_{t}\right)^{-1}
$$

where $\omega \equiv \omega_{r}+i \gamma, \nu_{f}$ is an effective collision frequency (negligible in this case), $\kappa$ is a continuous (Fourier-transform-type) dimensionless variable going from $-\infty$ to $\infty$, and $\omega_{t}$ is the transit frequency. Keeping only the resonant part, $G_{t} \simeq-\left.i \pi i \omega_{t}\right|^{-1} \delta\left(\kappa-\omega / \omega_{t}\right)$. The local magnetic drift frequency $\omega_{d}(\theta)$ enters in determining the weighting of the different $\kappa$ values for untrapped particles. ${ }^{\perp}$ For purposes of illustration only, making many unjustified approximations, the simplest ideal MHD ballooning mode equation, ${ }^{19}$ including an 
untrapped- $\alpha$-particle transit frequency resonance contribution, assuming $n_{a} \ll n_{i} \simeq n_{e}$ and $\beta \ll 1$ is:

$$
\begin{aligned}
\frac{d}{d \theta}\left[1+(s \theta-\alpha \sin \theta)^{2}\right] \frac{d \phi}{d \theta} & \div \alpha\{\cos \theta+(\hat{s} \theta-\alpha \sin \theta) \sin \theta] \phi \\
& +\frac{\omega\left(\omega-\omega_{\text {spi }}\right)}{\omega_{A}^{2}}\left[1+(\hat{s} \theta-\alpha \sin \theta)^{2}\right] \phi-i R_{\text {to }}=0
\end{aligned}
$$

for the "s- $\alpha$ " model MHD equilibrium, ${ }^{15}$ where $\omega_{* p i} \equiv \omega_{* i}\left(1-\eta_{i}\right)$. In fact, the results presented in this paper are obtained by solving much more comprehensive and accurate equations, presented in detail in Ref. 1. From the equations of Ref. 1, after some algebra, it can be shown that the untrapped- $\alpha$-particle transit-frequency resonance term $R_{t a}$ is approximately

$$
R_{\mathrm{l \alpha}} \simeq \frac{\omega_{\omega_{\alpha}} \bar{\omega}_{d \alpha}}{\omega_{A}^{2} k_{j}^{2} \rho_{i}^{2} \bar{\omega}_{l \alpha}} \frac{n_{\alpha}}{n_{e}} \frac{T_{i}}{T_{\alpha}} C
$$

where $C$ is a dimensionless function which can have either sign and which is of order unity for $\omega \sim \bar{\omega}_{t a}$. For $\omega \not \bar{\omega}_{t a}$, when $F_{\alpha}=F_{\text {ma }},|C|$ is of order $\left(\omega / \bar{\omega}_{t a}\right)^{2} \exp \left[-\left(\omega / \bar{\omega}_{t a}\right)^{2}\right]$; and when $F_{\alpha}=F_{S D},|C|$ is of order $\left(\omega / \bar{\omega}_{t a}\right)^{2} H\left(v_{\alpha}-\omega / \bar{\omega}_{t a}\right) /\left[\left(\omega / \bar{\omega}_{t a}\right)^{3}+\left(v_{c} / v_{t h \alpha}\right)^{3}\right]$, where $v_{t h \alpha} \equiv\left(2 T_{\alpha} / \pi_{\alpha}\right)^{1 / 2}$. Balancing the $R_{t a}$ term with the $\omega\left(\omega-\omega_{-p i}\right)$ term, the $R_{t a}$ contribution to the growth rate is of order:

$$
\frac{\gamma_{t a}}{\omega_{\alpha e}} \sim \frac{\omega_{\omega e}}{\bar{\omega}_{t i}} \frac{1}{k_{\theta}^{2} \rho_{i}^{2}} \frac{n_{\alpha}}{n_{e}}\left(\frac{T_{\alpha}}{T_{e}} \frac{m_{\alpha}}{m_{i}}\right)^{1 / 2}\left(\frac{T_{i}}{T_{e}}\right)^{3 / 2} \frac{r_{n e}^{2}}{r_{n a} R_{0}} C,
$$

which is of order unity for the parameters of Table III, where $\omega \sim \bar{\omega}_{t a}$, consistent with the numerical results of solving the more complete equations. The small value of $n_{a} / n_{e}=O\left(10^{-2}\right)$ is compensated by the small value of $\left(k_{p} p_{i}\right)^{2}=O\left(10^{-2}\right)$. The trapped- $\alpha$-particle bounce frequency resonance term would make a similar contribution, with a resonant denominator of the form ${ }^{1} G_{b} \equiv\left(\omega+i \nu_{f}-\omega_{d}^{(0)} \pm p w_{b}\right)^{-1}$, where $\omega_{d}^{(0)}$ is the trapped-particle-orbit time-average of the magnetic drift frequency $\omega_{d,} \omega_{b}$ is the bounce frequency, and $\mathrm{p}$ is summed over integer values. However, for the parameters of Table III, this trapped- $\alpha$ resonant contribution is smaller than that for untrapped $a$ 's, because of the greater mismatch between $\left|\omega_{r}\right|$ and $\bar{\omega}_{b a r}$. 
In contrast to the KMHDBM, the TEM has $\bar{\omega}_{d a} \simeq \bar{\omega}_{\mathrm{ca}}>\bar{\omega}_{b a} \gg \gamma \geqslant i \omega_{r}$ for the parameters in Table II, so the mode- $\alpha$-particle resonances are very weak.

A useful way of thinking about this a-particle effect on the kMHDBM is as follows: It is well known that the addition of resistivity, i.e., collisional dissipation, can cause small positive growth rates for the MHD baltooning mode even for $\beta<\beta_{c 1}^{\mathrm{MHD}}$. In the present case, collisions are negligible, but there is a much larger amount of $\alpha$-particle resonant dissipation which has the same effect.

The results of varying $\beta \propto n_{j}$ for fixed $T_{j}$ and $B_{0}$, in the manner already described for the TEM and the KMHDBM, are shown in Fig. 2 for the growth rate $\gamma$. Also shown in Fig. 2 is the growth rate curve for the simplest ideal MHD ballooning mode equation, without $\omega_{\text {spi }}$ and without $\alpha$-particles or helium, for the same magnetic surface in the same sequence of numerical MHD equilibria. Including $\omega_{-p i}$ and the many other kinetic effects, but with no $\alpha$-particles or helium, lowers the growth rate curves substantially to those for the kMHDBM, as has been seen before for varjous tokamaks in Refs. 20 and 18. The correspondisg kinetic $\beta$-critical, $\beta_{c 1}^{\text {kin }}$, can be either raised, as for $n=19$, or lowered, as for $n=58$, from the simple ideal MHD $\beta_{\mathrm{el}}^{\mathrm{MHD}}$, due to ion magnetic drift resonances and other kinetic effects. Including the $\alpha$-particles with $F_{\alpha}=F_{S D}$ and $\eta_{\alpha}=0$ for $n=19$ raises the grow'th rate substantially for $\beta<\beta_{c 1}^{\mathrm{MHD}}$ and, in fact, lowers $\beta_{c 1}^{\text {kin }}$ to below $2 \%$ ! This is a very substantial reduction whose implications will be discussed in Sec. IV. The addition of the helium ash for $\beta=\beta_{\mathrm{CIT}}=\mathbf{5 . 8 0 \%}$ has only a small additional destabilizing effect.

For the TEM, the growth rate curve without the $\alpha$-particles or helium is fairly insensitive to $\beta$-variation, at least out to $\beta=11.3 \%$. Adding the $\alpha$-particles or helium ash has only a slight stabilizing or destabilizing effect, respectively, for $\beta=\beta_{\mathrm{CIT}}=5.80 \%$. The TEM is unstable down to $\beta=0$ (electrostatic limit), and has a growth rate larger than that of the kMHDBM over this entire range of $\beta$. 
The corresponding real frequencies $w_{\text {, }}$ are shown in Fig. 3. They are all in the ion diamagnetic direction. The real frequency for the simple ideal MHD ballooning mode without $\omega_{\text {.pi }}$ is just $\omega_{r}=0$. For the k.MHDBM for $n=19$, adding the $\alpha$-particles is seen to substantially increase $\omega_{\mathbf{r}} ;$. Various characteristic frequencies are shown on the vertical axis. For $\beta=\beta_{\mathrm{CIT}}=5.80 \%$ and $n=19$, the $\alpha$-particle frequencies are seen to obey the ordering given in Eq.(1). The curves for the kMHDBM for $n=19$ correspond to $\left|\omega_{r} / \omega_{A}\right| \simeq 0.5-0.7$ with the $\alpha$-particles and to $\left|\omega_{r} / \omega_{A}\right| \simeq 0.2$ without them, where $\omega_{A} \propto n_{j}^{-1 / 2} \propto \beta^{-1 / 2}$.

The particular way in which $\beta \equiv 8 \pi \sum_{j} n_{j} T_{j} / B_{0}^{2}$ varies can make a difference. The results shown in Figs. 2 and 3 for $\gamma$ and $\omega_{r}$ assumed that $n_{e}, n_{i}$, and $n_{a}$ all varied proportional to $\beta$, with $T_{e}, T_{i}, T_{a}$, and $B_{0}$ fixed. In Figs. 4 and 5 , this variation for the kMHDBM for $n=19$ is compared with that for the situstion in which $T_{e}, T_{i}$, and $n_{a}$ vary proportional to $B$, with $n_{e}, n_{i}, T_{a}$, and $B_{0}$ fixed. This latter type of variation has some rough, qualitative correspondence to the way in which the parameters vary in time within the igaition simulation ${ }^{17}$ with BALDUR for CIT. In Fig. 4 for $\gamma$, the two curves with $\alpha$-particles cross at $\beta=\beta_{\mathrm{CIT}}$, where all of the parameters are the same. For $\beta \neq \beta_{\mathrm{CIT}}$, the variation of $\gamma$ is somewhat different, but the critical (local) $\beta$ values with the $\alpha$-particles are both about $2 \%$. The two corresponding curves without $\alpha$-particles are also shown. In Fig. 5 , the results for

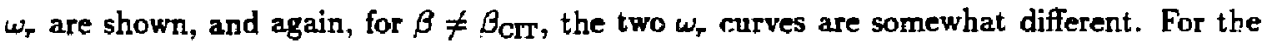
case with $n_{e}, n_{i}, n_{\alpha} \propto \beta$, where $\omega_{A} \propto \beta^{-1 / 2},\left|\omega_{r} / \omega_{A}\right|$ stays in the relatively narrow range from 0.5 to 0.7 , while in the case with $T_{e}, T_{i}, \pi_{\alpha} \propto \beta$, where $\omega_{A}$ is essentially constant, $\mid \omega_{r} / \omega_{A}$ i raries over a wider range from 0.5 to 0.9 .

The variations of $\gamma$ and $\omega_{r}$ with $k_{n} \rho_{i} \propto n$ are shown in Figs. 6 and 7 , respectively. The growth rate cuive for the TEM is broad, while those for the KMHDBM can be either broad or narrow, depending on the $\beta$-values and the particle species included. The $n$ values chosen for use in Figs. 2 and 3 are indicated in Fig. 6; they are at or near the growth rate maxima in $\pi$. In Fig. 7, the real frequencies are again all in the ion diamagnetic direction. 
The variation of $\gamma$ and $\omega_{r}$ with $n_{a} / n_{c}$ is shown in Fig. 8 for $\beta=\beta_{\mathrm{CTT}}=5.82 \%, n=19$. $F_{a}=F_{S D}$, and $\eta_{a}=0$, with no helium ash. The dependence of $\gamma$ and $\omega$, on $n_{\alpha} / n_{e}$ is seen to be almost linear, with a threshold at $n_{\mathbf{a}} / n_{e} \simeq 0.18 \%$. For higher $\beta$ values, the threshoid in $n_{\alpha} / n_{e}$ would be lower, reaching zero at $\beta=\beta_{\mathrm{cl}}^{\text {kin }}$.

The variation of $\gamma$ with $T_{\mathrm{a}}$ for the kMHDBM is shown in Fig. 9. Here, $T_{\mathrm{e}}$ and $T_{\mathrm{i}}$ are held fixed, with $F_{a}=F_{\max } \eta_{\mathrm{a}}=0, \beta=\beta_{\mathrm{CIT}}=5.82 \%$, and $n=22$, with no helium ash. This is a somewhat artificial quantity to vary, in that $F_{m \alpha}$ is used mainly as an approximation to the real $F_{\alpha}$, which should be close to $F_{S D}$. The $T_{\alpha}$ for $F_{\alpha}=F_{S D}$ is determined mainly by the $\alpha$ particle birth energy, $E_{\mathrm{a}}=3.52 \mathrm{MeV}$, and also by $v_{\mathrm{c}} \propto T_{e}^{1 / 2}$, and should not vary greatly in realistic situations. However, this rariation of $T_{a}$ is of interest with regard to understanding the $\alpha$-particle resonance mechanisms discussed previously. As $T_{\mathrm{a}}$ varies, different values of $\kappa$ and $p$, for untrapped and trapped $\alpha$-particles, respectively, will come into strongest resonance, since $\kappa \omega_{t}, p \omega_{b} \propto E^{1 / 2}$ and $\omega_{d}^{(0)} \propto E$, and the average energy $\langle E\rangle \equiv(3 / 2) T_{a}$. Some rather complicated, multipeaked structure of $\gamma$ with $T_{\alpha}$ is possible in this situation, and is seen in the numerical results in Fig. 9. Thus, this variation is consistent with the explanation of the destabilization of the kMHDBM by the $\alpha$-particle resonances.

Quasilinear transport coefficients for each species for particles, $D_{j}$, and for energy, $\kappa_{\boldsymbol{j}}$, calculated as described in Ref. 18, are shown in Table IV. Since a strictly quasilinear calculation does not predict the saturation level for the mode and thus does not predict :he absolute level of anomalous transport, but only the relative levels for particles and for energy and for the different plasma species, the results are given normalized to the energy diffusion coefficient for the electrons for each mode, $\kappa_{e}$, since :' is experimentally the best known of the coefficieric: The particle diffusion coefficients for each mode are automatically ambipolar, i.e., no net electric charge is transported, since the modes satisfy the quasineutrality cundition. 
For both modes, electron, ions, and thermalized helium ash particles are transported at comparable rates, within a factor of five or so, that is, all of them are transported anomalously, relative to neoclassjcal diffusion rates. The anomalous transport of helium ash, in particular, can be of benefit in a tokamak reactor. However, with respect to the transport of the hot $\alpha$-particles, there are major differences in the results for the two modes. The trapped-electron drift mode does not transport $\alpha$-particles, at any significant level, while the kinetically calculated MHD ballooning mode does. This is a result of the very weak resonant interaction of the $\alpha$-particles with the TEM, dur to the large difference in magnitude between the TEM mode frequency and the $\alpha$-particle bisunce, transit, and magnetic drift frequencies. Thus, if the tokamak were to operate at a low ep -ugh $\beta$ value that the KMHDBM was stable, but the TEM was unstable, then the thermalized helium ash would be transpotted out anomalcusly, while the $\alpha$-particles would he transported out only neoclassically, which is a desirable situation.

\section{TFTR}

The TFTR device should also be capable of producing significant numbers of $\alpha$-particles. A BALDUR transport code run for a situation designed to maximize the number of $\alpha$ particles, using a transport model that has reproduced experimental results relatively well in some cases, is described in Ref. 21. Choosing a time when the plasma parameters are not changing significantly, at the end of the neutral beam pulse, and evaluating the local parameters on a magnetic surface with $r / a \simeq 1 / 2$, the parameters that will be used for the TFTR case are: $\beta=\beta_{\text {TFTR }} \equiv \beta_{c}+\beta_{i}+\beta_{k \hbar}=1.44 \%, q=1.13, \hat{s}=0.727, \epsilon_{0}=0.185$, $r_{n e} / r=0.749, R_{0}=273 \mathrm{~cm}, B_{0}=5 \mathrm{~T}, I_{p}=3 \mathrm{MA}, \nu_{s}^{e}=0.0027$, and $n_{e}=2.27 \times 10^{13} \mathrm{~cm}^{-3}$. Other local parameters for the four species are given in Table $V$. To put the parameters into perspective, note that

$$
\frac{n_{a}}{n_{e}} \simeq 0.3 \%, \frac{\beta_{a}}{\beta_{\text {TFTR }}} \simeq 8 \%, \frac{\beta_{a}^{\prime}}{\beta_{\text {TFTR }}^{\prime}} \simeq 13 \%
$$


while

$$
\frac{n_{\text {He }}}{n_{e}} \simeq 0.4 \%, \frac{\beta_{\text {He }}}{\beta_{\mathrm{TFTR}}} \simeq 0.3 \%, \frac{\beta_{\text {He }}^{\prime}}{\beta_{\mathrm{TFTR}}^{\prime}} \simeq 0.2 \% \text {. }
$$

The results for $\gamma$ for varying $\beta \propto \Omega_{j}$, for fixed $T_{j}$ and $B_{0}$, are shown in Fig. 10 , corresponding to Fig. 2 for the CIT case. For the TEM, the growth rate drops gradually with $\beta$ from its maximum at $\beta=0$, but is still unstable at $\beta=8.89 \%$. The $\alpha$-particles with $F_{\alpha}=F_{S D}$ and the helium ash have only a very slight stabilizing effect on this mode, again because the mode frequency is so much smaller than the $\alpha$-particle characteristic frequencies. The critical $\beta$ value from the simplest ideal MHD ballooning mode calculation is $\beta_{c 1}^{\mathrm{MHD}}=2.98 \%$. With the kinetic corrections, but without $\alpha$-particles or helium, the kMHDBM for $n=16$ is unstable for $\beta>\beta_{c l}^{\text {hin }}=3.38 \%$. Including the $\alpha$-particles with $F_{\alpha}=F_{S D}$ is destabilizing for $\beta \leqq 4 \%$ and stabilizing for $\beta \geqq 4 \%$. The amount of stabilization for $\beta=4.42 \%$, say, is consistent with the estimate of Eq. (5) for $|C| \leqslant 1$, which is the expected magnitude of $|C|$ since $\left|\omega_{r}\right| \leqslant \bar{\omega}_{L \alpha}$ in this case. The a-particle destabilization is sufficient to decrease the critical $\beta$ to $2.92 \%$, but the amount of damping from the background species is too great for the $\alpha$-particles to push the critical $\beta$ much below $\beta_{c 1}^{\mathrm{MHD}}$, as occurred in the CIT case in Sec. II, so the $\mathrm{kMHDBM}$ is stable for $\beta=\beta_{\mathrm{TFTR}}=1.44 \%$. This is due in part to the low value of $T_{e} / T_{i}$ for the TFTR case, $T_{c} / T_{i}=0.201$, compared to $T_{\varepsilon} / T_{i}=0.974$ for the CIT case. For instance, if $T_{e}$ and $d T_{e} / d r$ are artificially increased so that $T_{e} / T_{i}=1$ for the TFTR case (at fixed $\beta$ and $d \beta / d r$, so that the $n_{j}$ and $d n_{j} / d r$ decrease), the critical $\beta \propto n_{j}$ value with the $\alpha$-particles decreases from $2.92 \%$ to $2.07 \%$. The effect of the helium ash is very slightly stabilizing for this mode.

The corresponding real frequencies for the TEM and the kMHDBM are shown in Fig. 11. They are all in the ion diamagnetic direction. The real frequency for the simple ideal MHD ballooning mode without $\omega_{\text {-pi }}$ is just $\omega_{r}=0$. For the $k M H D B M$ for $n=16$, adding the $\alpha$-particles increases $\left|\omega_{r}\right|$ somewhat. Various characteristic frequencips are shown on the vertical axis. For $\beta \geq \beta_{c 1}^{M H D}$ and $n=16$, the $\alpha$-particle frequencies again obey the ordering 
given in Eq. (1). The results for $\omega_{r}$ for the $\mathrm{kMHDBM}$ for $n=16$ give $\left|\omega_{r} / \omega_{d}\right| \simeq 0.7$ with or without the $\alpha$-particles, where $\omega_{A} \propto n_{j}^{-1 / 2} \propto \beta^{-1 / 2}$.

The variation of $\gamma$ with $k_{\theta} \rho_{i} \propto n$ is shown in Fig. 12 for the TEM at $\beta=\beta_{\text {TFTR }}=1.44 \%$ and for the kMHDBM at $\beta=4.42 \%$ without $\alpha$-particles or helium ash. The growth rate curve for the TEM is very broad around the maximum at $n=\mathbf{5 0}$ or $k_{\theta} \rho_{i}=0.63$, while the curve for the kMHDBM is narrower around the maximum at $n=16$ or $k_{\theta} \rho_{i}=0.16$. The $n$ values chosen for use in Figs. 10 and 11 are indicated in Fig. 12. They are at or near the growth rate maxima in $\pi$. The corresponding real frequencies are all in the ion diamagnetic direction.

Quasilinear transport coefficients are given in Table VI, corresponding to those in Table IV for the CIT case. In this TFTR case, for both the TEM at $\beta=\beta_{\text {TFTR }}=1.44 \%$ and the kMHDBM at $\beta=4.42 \%$, the diffusion coefficients for ions and electrons are comparable, but those for $\alpha$-particles and helium ash are negligible. This is in contrast to the CIT case, where both modes transported helium ash at a significant level, and the KMHDBM transported $\alpha$-particles at a significant level.

\section{CONCLUSIONS}

Results have been presented for the trapped-electron drift mode and the kinetically calculated MHD ballooning mode for cases representative of CIT and TFTR, from a relatively complete high- $\pi$ linearized kinetic calculation. In particular, this calculation retains the resonances of the mode with the trapped-particle bounce frequency, the untrapped-particle transit frequency, and the magnetic drift frequency for each species. The effects of the hot a-particles and the thermalized helium ash particles on the mode growth rates and real frequencies, and the relative quasilinear particle and energy transport coefficients for the different plasma species have been investigated.

For both the CIT and TFTR cases, the effect of the hot $a$-particles and the thermalized helium ash particles on the trapped-electron drift mode is weak, due to the large difference 
in magnitude between the mode real frequency and the single- $\alpha$-particle characteristic frequencies. Also, the effect of the helium ash particles on the kinetically calculated MHD ballooning mode is weak. On the other hand, the hot $\alpha$-particles can have an important effect on this mode. Their effect is destabilizing for (local) $\beta$ below a "crossover" value $\beta^{\mathrm{c}}$, and stabilizing for $\beta$ above $\beta^{\mathrm{cr}}$, where $\beta^{\mathrm{cr}}$ is somewhat larger than the simplest ideal MHD critical $\beta, \beta_{\mathrm{cl}}^{\mathrm{MHD}}$. For the CIT case, where the damping of the background electrons and ions is not too strong, the $\alpha$-particle destabilization is sufficient to lower the critical $\beta$ value for the kinetically calculated MHD ballooning mode to about $2 \%$, substantially below $\beta_{c 1}^{\mathrm{MHD}} \simeq 8 \%$.

This a-particle destabilization in the CIT case is mainly due to the transit-frequency resonances of the untrapped $\alpha$-particies, with smaller contributions from the bounce-frequency resonances and the magnetic drift frequency resonances of the trapped $\alpha$-particles. The transit-frequency resonances are strong because of the near coincidence of the mode real frequency and the average transit frequency of the untrapped $\alpha$-particles in the CIT case. In the TFTR case, the amount of $\alpha$-particle destabilization is roughly comparable to that in the CIT case, but the damping from the background electrons and ions is stronger, so that the critical $\beta$ with the $\alpha$-particles, $\beta_{\mathrm{cI}}^{\alpha}$, is not much below the simplest ideal MHD critical $\beta$, $\beta_{c 1}^{\mathrm{MHD}} \simeq 3 \%$.

The relative quasilinear transport coefficients for particles and energy for each plasma species have also been calculated. For the CIT case, the thermalized helium ash is transported by both the TEM and the KMHDBM at a level roughly comparable to those of the background electrons and ions, while the hot $\alpha$-particles are transported at a significant level only by the KMHDBM, not by the TEM. For the TFTR case, on the other hand, the transport of both the helium ash and the hot $\alpha$-particles is negligible for both the TEM and the kMHDEM. Thus, the results for anomalous transport turn out to be quite case dependent.

Anomalous transport of the thermalized helium ash, if jt occurs, is a desirable thing, limiting accumulation of helium ash in the center of the discharge. Conversely, anomalous 
transport of the hot $\alpha$-particles, which can remove them from the center of the discharge before they transfer all of their energy to heat the background species, is undesirable. However. the hot $\alpha$-particle slowing-down time in CIT is still expected to be considerably shorter than the anornalous $\alpha$-particle loss time, ${ }^{22}$ so that the change in the power balance is not major. $A$ more serious potential effect is $\beta$-saturation at a lower value than that needed to sustain ignition in this specific design for CIT, due to the substantial reduction in the critical $\beta$ for the MHD ballooning mode from the a-particle destabilization. It should be pointed out, though, that the trapped-electron drift mode has a higher linear growth rate than the MHD ballooning mode, even with the $\alpha$-particle destabilization, throughout the range $0 \leq \beta \leq \beta_{c 1}^{\mathrm{MHD}}$, and that the nonlinear consequences in this situation for the MHD ballooning mode are not well understood. The relative quasilinear diffusion coefficients presented earlier assumed that the two modes acted independently, and this may be too pessimistic an assumption.

A number of interesting possibilities arise for future work $i$ the area of $\alpha$-particle effects on tokamak instabilities. The present work has looked exclusively at the effects of $\alpha$-particles on preexisting modes, i.e., those which can be unstable even without the $\alpha$-particles. There is also the possibility that the a-particles could give rise to additional roots of the dispersion equations, above some threshold in $n_{a}$ or $\beta_{\alpha}$. The present work has also looked exclusively at cases where $F_{\alpha}$ is uniform in pitch angle. In the presence of loss regions in pitch angle, ${ }^{16}$ however, $F_{a}$ would become nonuniform in pitch angle, leading to additional terms in the mode equations. ${ }^{23.24}$ The nonuniformity could have interesting effects on the low frequency modes considered here, but it could also destabilize ion and $\alpha$-particle cyclotron frequency modes. Also, while the present calculation includes full finite Larmor radius effects for all species, it does not include full finite banana width effects, which could have an additional stabilizing effect. 


\section{ACKNOWLEDGMENTS}

The author would like to thank Drs. D. P. Stotler, M. H. Redi, and G. Bateman for providing the BALDLR transport code results, Drs. M. H. Hughes and A. M. M. Todd for providing the numerical MHD equilibria, and Drs. Liu Chen, G. W. Hammett, H. E. Mynnick. D. J. Sigmar, W. M. Tang, R. B. White, and S. J. Zweben, for informative discussions. This work was supported by United States Department of Energy Contract No. DE-AC02-76CHO-3073. 


\section{REFERENCES}

'G. Rewoldt, W. MI. Tang, and M. S. Chance, Phys. Fluids 25, 480 (1982).

${ }^{2}$ See National Technical Information Service Document No. PPPL-2480, by H. P. Furth (Princeton L̈niversity Plasma Physics Laboratory Report PPPL-2480, 1987). Copies may be ordered from the National Technical Information Service, Springfield, Virginia 22161. The price is $\$ 7.00$ plus a $\$ 3.00$ handling fee. All orders must be prepaid.

${ }^{3}$ S. J. Zweben, Physica Scripta T16, 119 (1987).

${ }^{4}$ B. Coppi and F. Pegoraro, Ann. Phys. 134, 376 (1981).

${ }^{5}$ D. A. Spong, D. J. Sigmar, W. A. Cooper, and D. E. Hastings, Phys. Fluids 28, 2494 (1985).

${ }^{6}$ See National Technical Information Service Document No. ORNL/TM-9688, by D. A. Spong, D. E. Hastings, D. J. Sigmar, and W. A. Cooper, (Oak Ridge National Laboratory Technical Memorandum 9688, 1985). Copies may be ordered from the National Technical Information Service, Springfield, Virginia 22161. The price is $\$ 7.00$ plus a $\$ 3.00$ handling fee. All orders must be prepaid.

${ }^{\text {T}}$ D. A. Spong, D. J. Sigmar, K. T. Tsang, J. J. Ramos, D. E. Hastings, and W. A. Cooper, Physica Scripta T16, 18 (1987).

${ }^{8}$ D. A. Spong, D. J. Sigmar, and J. J. Ramos, Fusion Technol. 13, 428 (1988).

${ }^{9}$ J. J. Ramos, D. A. Spong, and D. J. Sigmar, Bull. Am. Phys. Soc. 32, 1771 (1987).

${ }^{10} \mathrm{~J}$. Weiland and Liu Chen, Phys. Fluids 28, 1359 (1985), and references therein.

${ }^{11}$ Liu Chen and R. B. White, Bull. Am. Phys. Soc. 32, 1771 (1987).

${ }^{12}$ C. E. Singer, D. E. Post, D. R. Mikkelsen, M. H. Redi, A. McKenney, A. Silverman, F. G. P. Seidl, P. H. Rutherford, R. J. Hawryluk, W. D. Langer, L. Foote, D. B. Heifetz, W. A. Houlberg, M. H. Hughes, R. V. Jensen, G. Lister, and J. Ogden, Comp. Phys. Comm. (in press).

${ }^{13}$ J. D. Gaffey, Jr., J. Plasma Phys. 16, part 2, 149 (1976). 
${ }^{14}$ J. L. Johnson, H. E. Dahied, J. M. Greene, R. C. Grimm, Y. Y. Hsieh, S. D. Jardin. J. Manickam, M. Okabayashi, R. G. Storer, A. M. M. Todd, D. E. Voss, and K. E. Weimer, J. Comp. Phys. 32, 212 (1979).

15.J. W. Connor. R. J. Hastie, and J. B. Taylor, Phys. Rev. Lett. 40, 396 (1978).

${ }^{16}$ R. B. White, S. J. Zweben, M. S. Chance, H. E. Mynick, and W. M. Nevins, Bull. Am. Phys. Soc. 32, 1918 (1987).

${ }^{17}$ See National Technical Information Service Document No. PPPL-2510, by D. P. Stotler and G. Bateman (Princeton University Plasma Physics Laboratory Report PPPL-2510, 1988). Copies may be ordered from the National Technical Information Service, Springfield, Virginia 22161 . The price is $\$ 7.00$ plus a $\$ 3.00$ handling fee. All orders must be prepaid.

${ }^{18}$ G. Rewoldt, W. M. Tang, and R. J. Hastie, Phys. Fluids 30, 807 (1987).

${ }^{19}$ W. M. Tang, J. W. Connor, and R. B. White, Nucl. Fusion 21, 891 (1981).

${ }^{20}$ W. M. Tang, G. Rewoldt, C. Z. Cheng, and M. S. Chance, Nucl. Fusion 25, 151 (1985).

${ }^{21}$ See National Technical Information Service Document No. PPPL-2535, by S. J. Zweben, M. H. Redi, D. R. Mikkelsen, and J.D. Strachan, (Princeton University Plasma Physics Laboratory Report PPPL-2535, 1988). Copies may be ordered from the National Technical Information Service, Springfield, Virginia 22161 . The price is $\$ 7.00$ plus a $\$ 3.00$ handling fee. All orders must be prepaid.

${ }^{22} \mathrm{G}$. $W$. Hammett, private communication.

${ }^{23}$ P. J. Catto, W. M. Tang, and D. E. Baldwin, Plasma Phys. 23, 639 (1981).

${ }^{24}$ G. Rewoldt and W. M. Tang, Phys. Fluids 26, 3619 (1983). 
TABLE I. Local parameters for plasma species for the CIT case.

\begin{tabular}{lllllll}
\hline \hline$j$ & $Z_{j}$ & $m_{j}(\mathrm{amu})$ & $n_{j} / n_{\mathrm{e}}$ & $T_{j}(\mathrm{keV})$ & $\eta_{j}$ & $r_{n j} / r_{n e}$ \\
\hline$e$ & -1 & $1 / 1836$ & 1 & 18.9 & 1.28 & 1 \\
$i$ & 1 & 2.5 & 0.817 & 19.4 & 1.56 & 1.165 \\
$\alpha$ & 2 & 4 & 0.00532 & $904 .^{\mathrm{a}}$ & $0.118^{\mathrm{a}}$ & 0.340 \\
$\mathrm{He}$ & 2 & 4 & 0.0860 & 19.4 & 0.903 & 0.671 \\
\hline \hline
\end{tabular}

- These values for $T_{a}$ and $\eta_{a}$ are from the BALDUR results and are used for $F_{a}=F_{m a}$. Far $F_{\alpha}=F_{S D}$, the values $T_{\alpha}=942 \mathrm{keV}$, calculated from the slowing-down distribution, and $\eta_{L}=0$ are etnployed.

TABLE II. $\alpha$-particle and helium effects on linear eigenfrequencies $\omega$ for the trapped-electron drift mode (TEM) and the kinetically calculated MHD ballooning mode (kMHDBM) in units of $\omega_{\text {se }}$ for the CIT parameters.

\begin{tabular}{|c|c|c|c|c|c|}
\hline$\overline{\text { mode }}$ & & & TEM & kMHDBM & kMHDBM \\
\hline$\beta$ & & & $5.82 \%$ & $5.82 \%$ & $11.3 \%$ \\
\hline$n$ & & & 58 & 22 & 22 \\
\hline$k_{\theta} \rho_{i}$ & & & 0.36 & 0.14 & 0.13 \\
\hline$j$ & $F_{\alpha}$ & $\eta_{\mathbf{a}}$ & $\omega / \omega_{* e}$ & $\omega / \omega_{\text {ee }}$ & $\omega / \omega_{\text {we }}$ \\
\hline$\overline{e, i}$ & - & - & $-0.249+0.358 i$ & (damped) & $-2.52+0.726$ \\
\hline$e, i, \alpha$ & $F_{m a}$ & 0.118 & $-0.215+0.333 i$ & $-9.50+0.401 i$ & $-5.76+0.577$ \\
\hline$e, i, \alpha$ & $F_{m a}$ & 0 & $-0.218+0.336 i$ & $-9.21+0.361 i$ & $-5.40+0.605$ \\
\hline$e, i, \alpha$ & $F_{S D}$ & 0 & $-0.218+0.336 i$ & $-9.27+0.597 i$ & $-5.48+0.793$ \\
\hline$e, i, H e$ & - & - & $-0.211+0.373 i$ & (damped) & $-2.35+0.615$ \\
\hline$e . i, \alpha, H e$ & $F_{S D}$ & 0 & $-0.169+0.340 i$ & $-11.6+0.638 i$ & $-5.62+0.781 i$ \\
\hline
\end{tabular}


TABLE IIl. Changes in growth rate $\gamma$ and real frequency $\omega_{r}$ from different $\alpha$-particle terms and effects, for $\beta=\beta_{C I T}=5.82 \%, n=19, k_{\theta} \rho_{i}=0.12, k_{\theta P_{\alpha}}=0.51, F_{\alpha}=F_{S D}$, and $\eta_{\alpha}=0$, with no helium.

\begin{tabular}{lll}
\hline \hline term & $\Delta y / w_{\text {ace }}$ & $\Delta w_{r} / w_{\text {ar }}$ \\
\hline trapped- $\alpha p=0$ (time-average) term & +0.071 & -0.83 \\
trapped- $\alpha p \neq 0$ (non-time-average) term & +0.122 & +0.03 \\
untrapped- $\alpha$ term & +0.363 & +0.94 \\
adiabatic term & -0.011 & +0.17 \\
$n_{1} / n_{\mathrm{c}}$ and $r_{\text {ni }} / r_{\text {ne }}$ changes & -0.108 & -1.62 \\
$\beta$ changes from $\alpha$ 's & +0.004 & +0.48 \\
\hline \hline
\end{tabular}

-To preserve (bulk) charge neutrality and its radial derivative.

TABLE IV. Relative quasilinear transport coefficients for $\beta=\beta_{\text {CrT }}=5.82 \%$ for the trapped-electron drift mode (TEM) and the kinetically calculated MHD ballooning mode (kMHDBM).

\begin{tabular}{lllll}
\hline \hline & \multicolumn{2}{c}{ TEM( $(n=58)$} & \multicolumn{2}{c}{$\operatorname{kMHDBM}(n=19)$} \\
$j$ & $D_{j} / \kappa_{e}$ & $\kappa_{j} / \kappa_{e}$ & $D_{j} / \kappa_{e}$ & $\kappa_{j} / \kappa_{e}$ \\
\hline$e$ & 0.33 & 1 & 0.33 & 1 \\
$i$ & 0.11 & 0.47 & 0.097 & 0.48 \\
$\alpha$ & 0.002 & -0.001 & 0.25 & 0.54 \\
$H e$ & 0.15 & 0.46 & 0.064 & 0.22 \\
\hline
\end{tabular}


TABLE V. Local parameters for plasma species for the TFTR case.

\begin{tabular}{lllllll}
\hline$j$ & $Z_{j}$ & $m_{j}(a m u)$ & $n_{j} / n_{e}$ & $T_{j}(\mathrm{keV})$ & $\eta_{j}$ & $r_{\mathrm{n} j} / r_{\text {rec }}$ \\
\hline$e$ & -1 & $1 / 1836$ & 1 & 5.25 & 0.922 & 1 \\
$i$ & 1 & 2.5 & 0.985 & 26.1 & 0.766 & 1.0026 \\
$\alpha$ & 2 & 4 & 0.00322 & $8144^{\circ}$ & $0.123^{\circ}$ & 0.411 \\
$H e$ & 2 & 4 & 0.00413 & 26.1 & 3.92 & 5.13 \\
\hline \hline
\end{tabular}

a These values for $T_{\alpha}$ and $\eta_{\alpha}$ are from the BALDUR results and are used for $F_{\alpha}=F_{\text {ma. }}$. For $F_{\alpha}=F_{S D}$, the values $T_{\alpha}=698 \mathrm{keV}$, calculated from the slowing-down distribution, and $\eta_{a}=0$ are employed.

TABLE VI. Relative quasilinear transport coefficients for $\beta=\beta_{\text {TFTR }}=1.44 \%$ for the trapped-electron drift mode (TEM) and for $\beta=4.42 \%$ for the kinetically calculated MHD ballooning mode (kMHDBM).

\begin{tabular}{lllll}
\hline \hline & \multicolumn{2}{c}{$\operatorname{TEM}(n=47)$} & \multicolumn{2}{c}{$\operatorname{kMHDBM}(n=16)$} \\
$j$ & $D_{j} / \kappa_{e}$ & $\kappa_{j} / \kappa_{e}$ & $D_{j} / \kappa_{e}$ & $\kappa_{j} / \kappa_{\mathrm{e}}$ \\
\hline$i$ & 0.40 & 1 & 0.31 & 1 \\
$i$ & 0.40 & 0.73 & 0.30 & 1.1 \\
$\alpha$ & 0.004 & 0.0004 & 0.01 & 0.01 \\
$\mathrm{He}$ & -0.0002 & 0.0001 & -0.00001 & 0.0001 \\
\hline \hline
\end{tabular}




\section{FIGURE CAPTIONS}

FIG. 1. Magnetic surfaces for the MHD equilibrium for the CIT case.

FIG. 2. Linear growth rates $\gamma$ versus (lacal) $\beta \propto n_{j}$, for fixed $T_{j}$ and $B_{0}$, for the CIT parameters, in units of $10^{5} \mathrm{sec}^{-1}$, for the trapped-electron drift mode (TEM) and the kinetically calculated MHD ballooning mode (kMHDBM). Results from the simplest ideal MHD ballooning mode equation with $w_{\text {upi }}=0$ are also shown for reference.

FIG. 3. Real frequencies $\omega_{r}$ corresponding to Fig. 2. Characteristic single-particle frequencies are indicated on the vertical axis. All real frequencies here are in the ion diamagnetic direction.

FIG. 4. Growth rates $\gamma$ versus (local) $\beta$ for the kMHDBM for $n=19$ for the CIT parameters. Results are shown for cases with $\alpha$-particles where $n_{e}, n_{i}, n_{\alpha} \propto \beta$, at fixed $T_{e}, T_{i}, T_{a}$, and where $T_{e}, T_{i}, n_{\alpha} \propto \beta$ at fixed $n_{e}, n_{i}, T_{\alpha}$. The corresponding curves without $\alpha$-particles are also shown.

FIG. 5. Real frequencies $\omega_{r}$ corresponding to Fig. 4. All real frequencies here are in the ion diamagnetic direction.

FIG. 6. Variation of $\gamma$ with $k_{\theta} \rho_{i} \propto n$ for the TEM and the kMHDBM for the CIT parameters. The $n$ values used in Figs. 2 and 3 are indicated in parentheses.

FIG. 7. Variation of $\omega_{r}$ with $k_{\rho} \rho_{i} \propto n$ corresponding to Fig. 4.

FIG. 8. Variation of $\gamma$ and $\omega_{r}$ with $n_{a} / n_{e}$ for the CIT parameters for $\beta=\beta_{\text {CrT }}=5.82 \%$, $n=19, F_{\alpha}=F_{S D}$, and $\eta_{\alpha}=0$, with no helium ash.

FIG. 9. Variation of $\gamma$ with $T_{a}$ for the kMHDBM for the CIT parameters with $T_{e}$ and $T_{i}$ held fixed, $F_{\mathrm{a}}=F_{\mathrm{ma}} \eta_{\mathrm{a}}=0, \beta=\beta_{\mathrm{CII}}=5.82 \%$, and $\mathrm{n}=22$, with no helium ash.

FIG. 10. Variation of $\gamma$ with (local) $\beta \propto n_{j}$, for fixed $T_{j}$ and $B_{0}$, corresponding to Fig, 2 , for the TFTR parameters. 
FIG. 11. Real frequencies $\omega_{r}$ corresponding to Fig. 10. Characteristic single-particle frequencies are indicated on the vertical axis. All real frequencies here are in the ion diamagnetic direction.

FIG. 12. Variation of $\gamma$ with $k_{\theta} \rho_{i} \propto n$ for the TEM and the kMHDbM for the TFTR parameters. The $n$ values used in Figs. 10 and 11 are indicated in parentheses. 


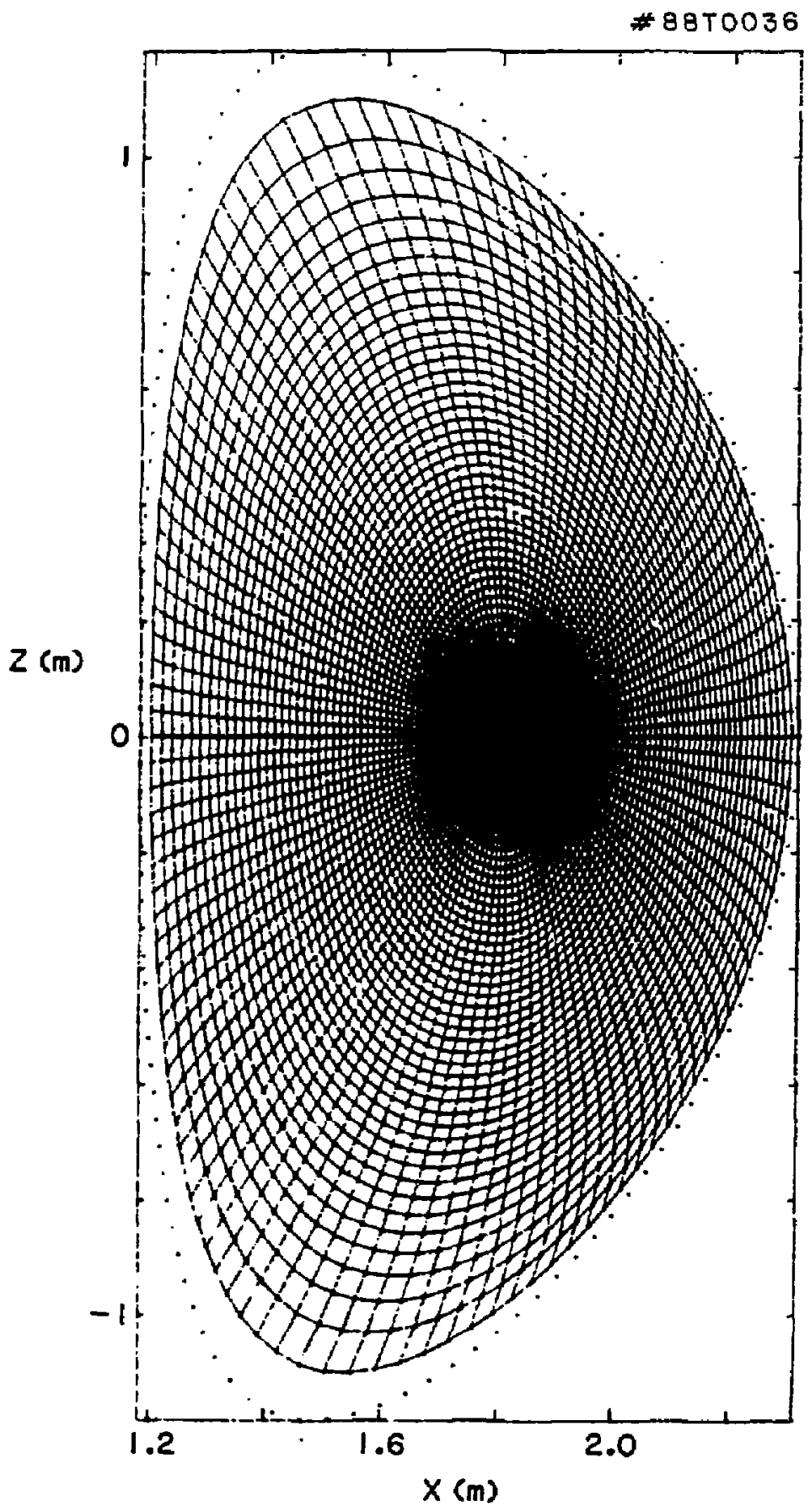

Fig. 1 


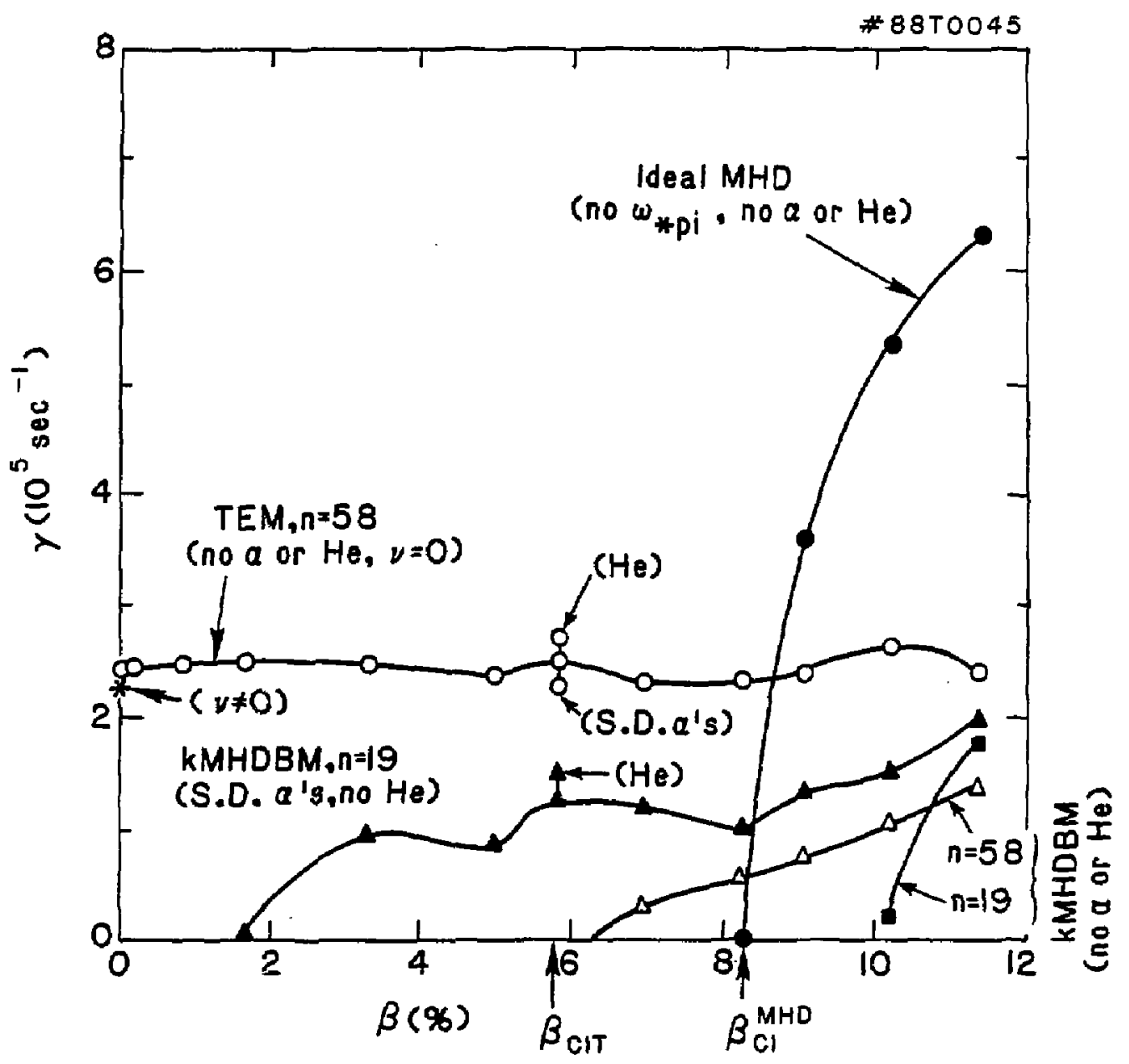

Fig. 2 


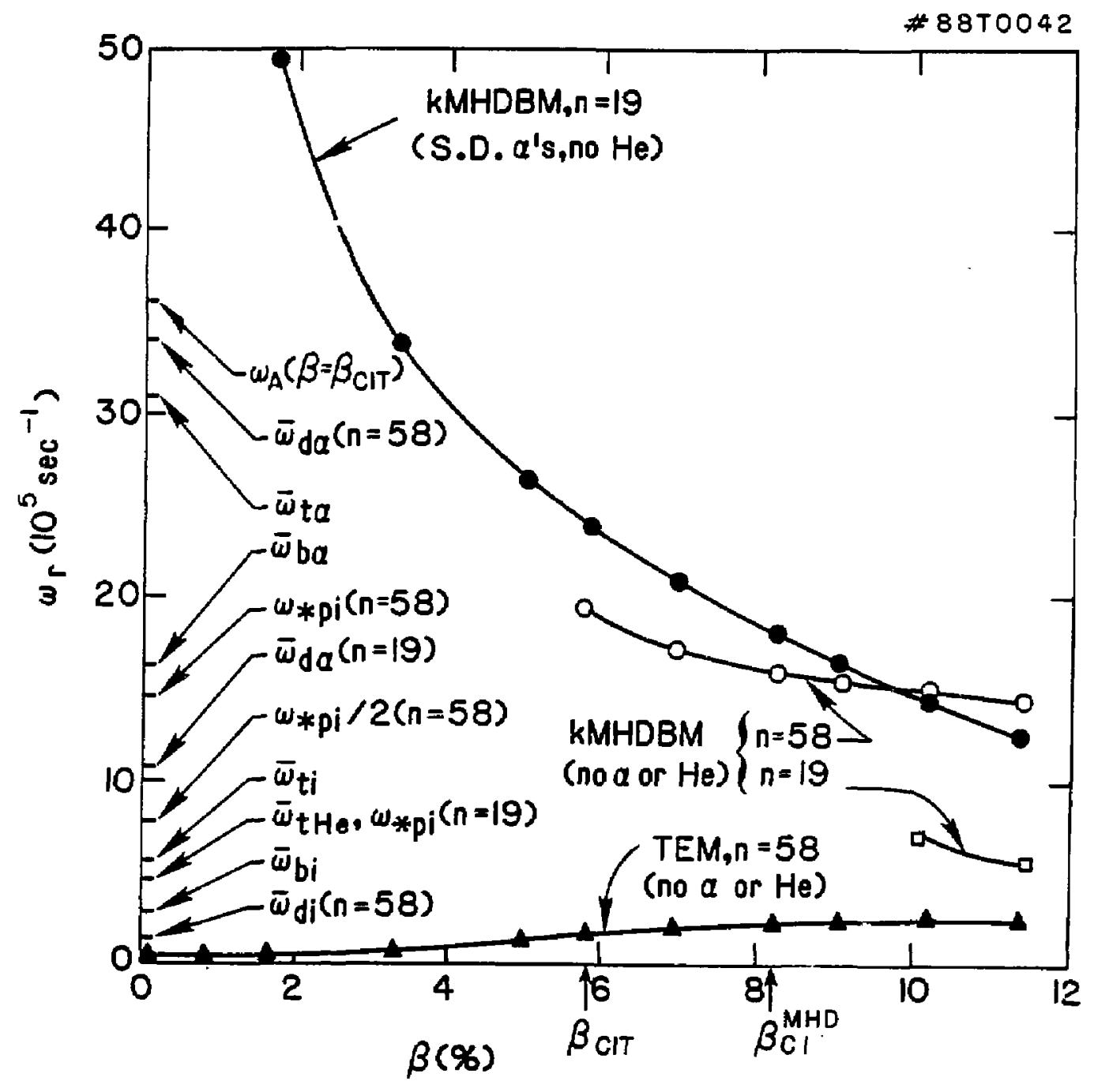

Fig. 3 


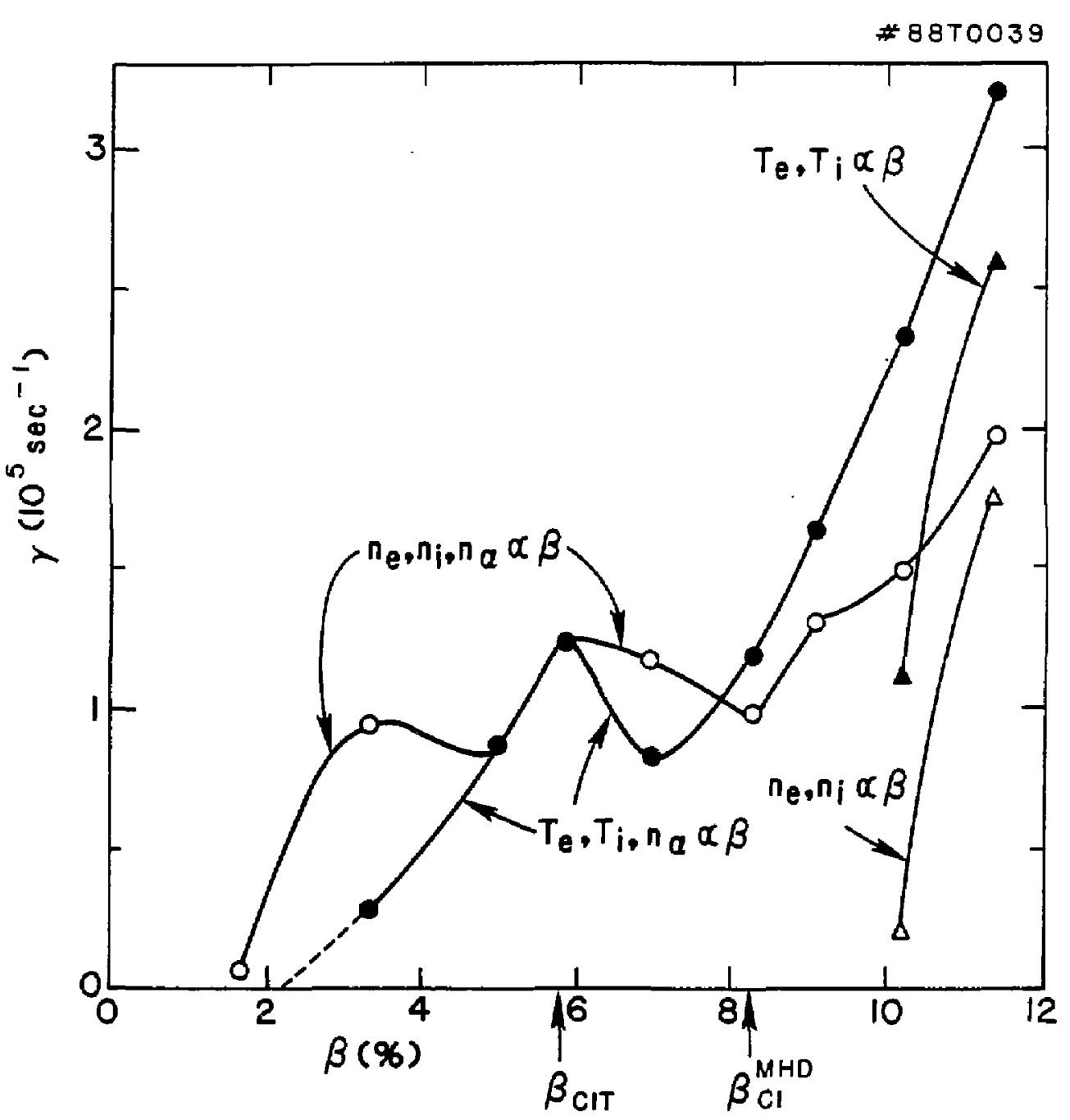

Fig. 4 


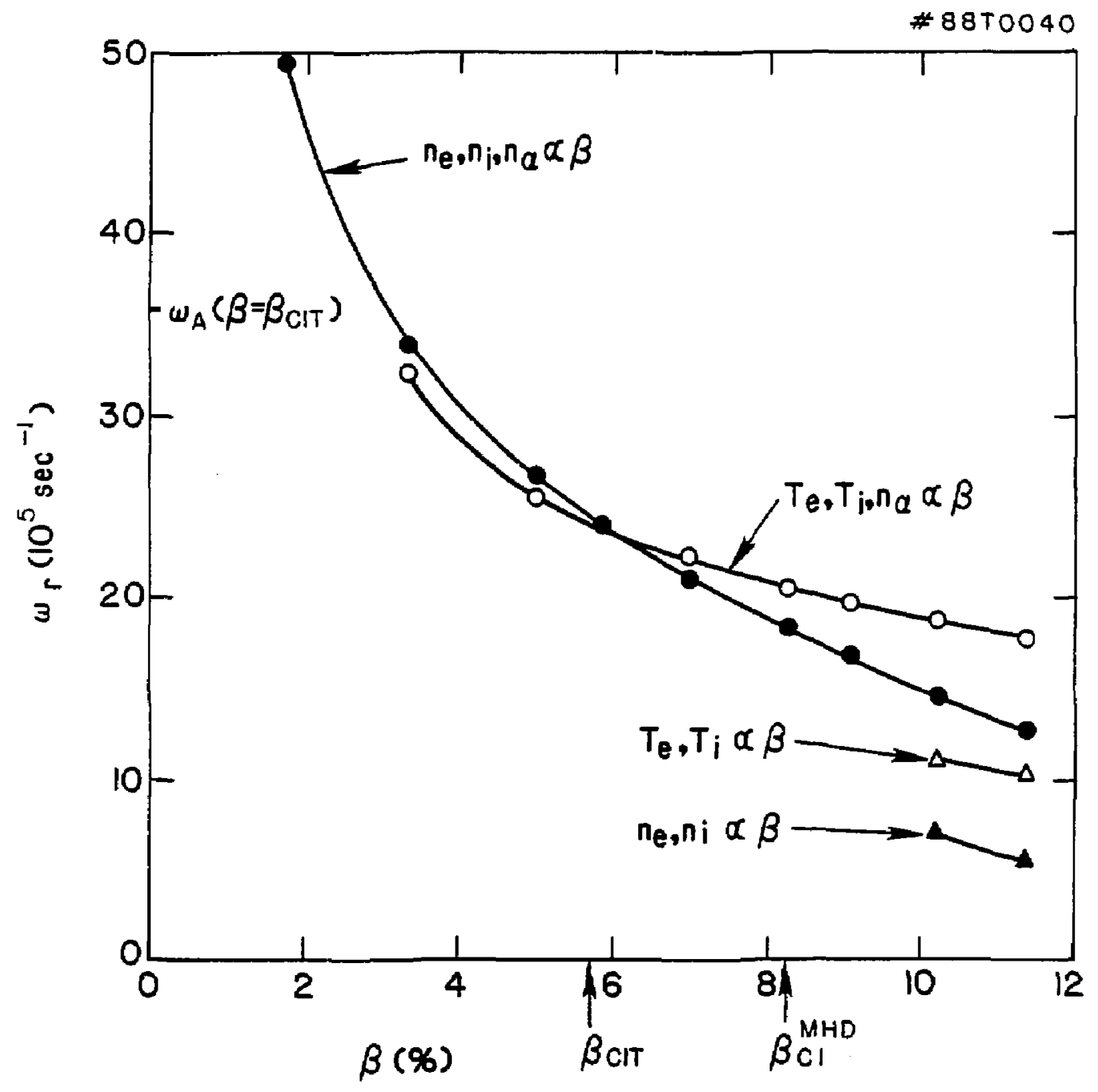

Fig. 5 


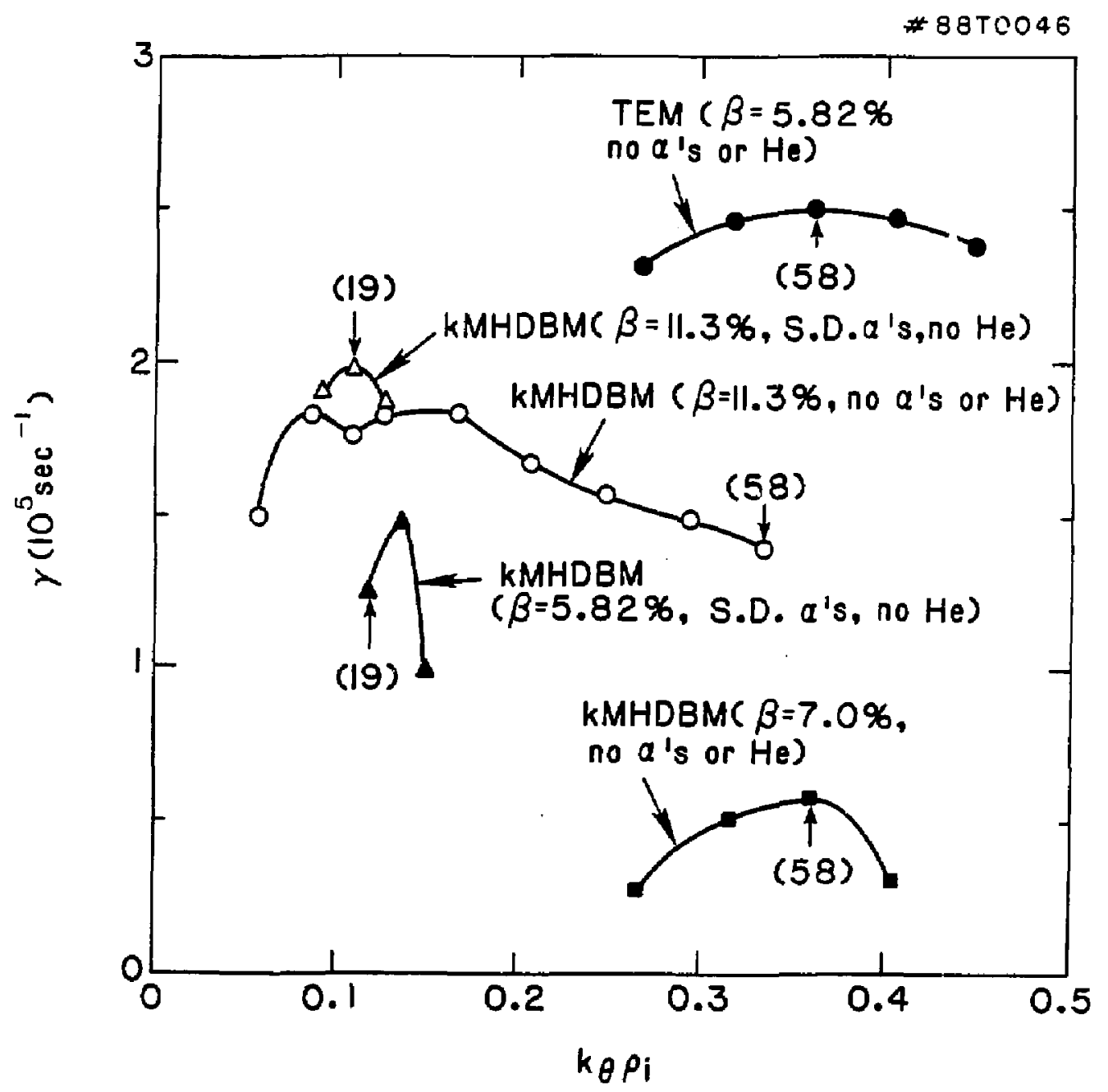

Fig. 6 


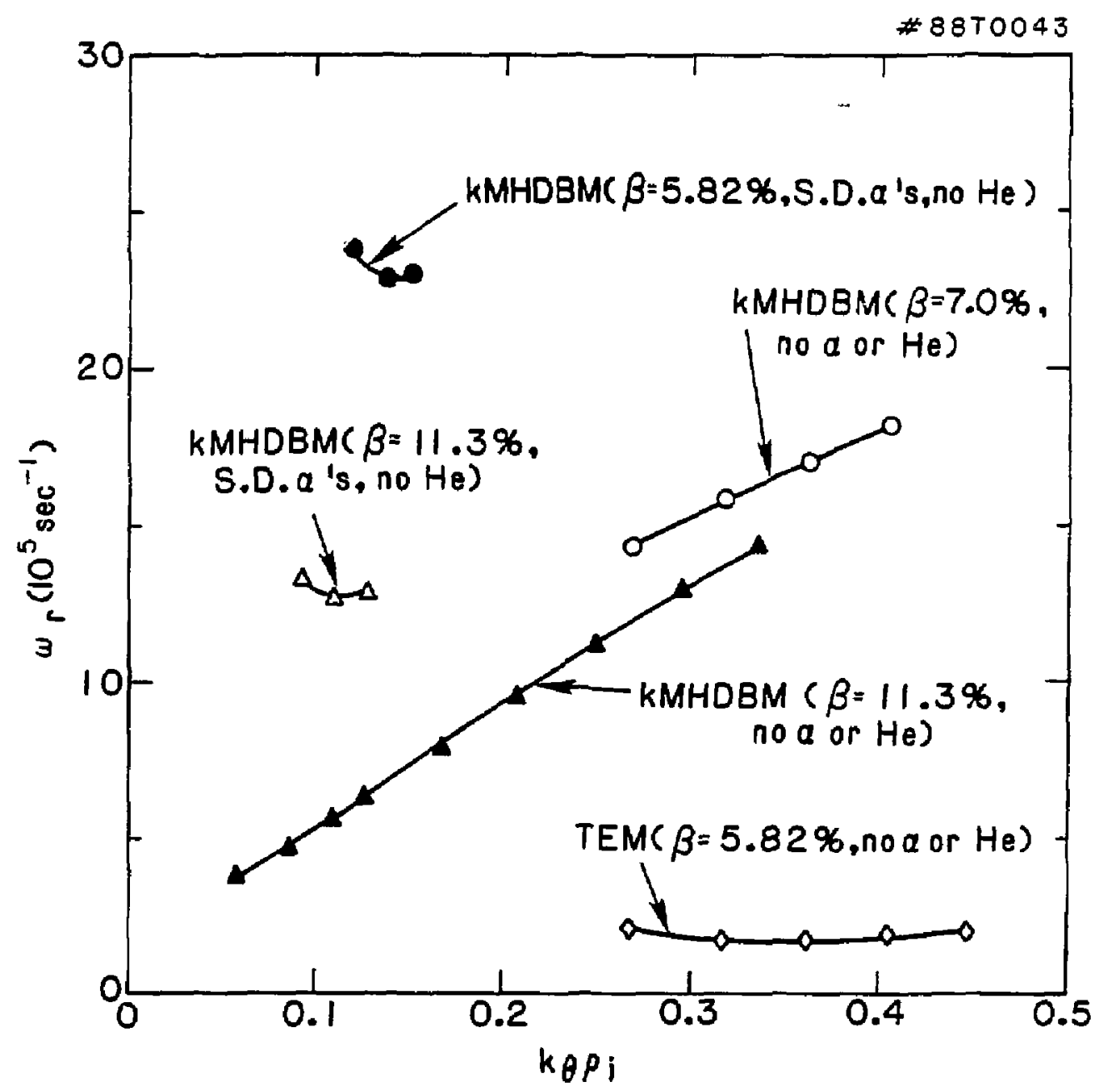

Fig. 7 


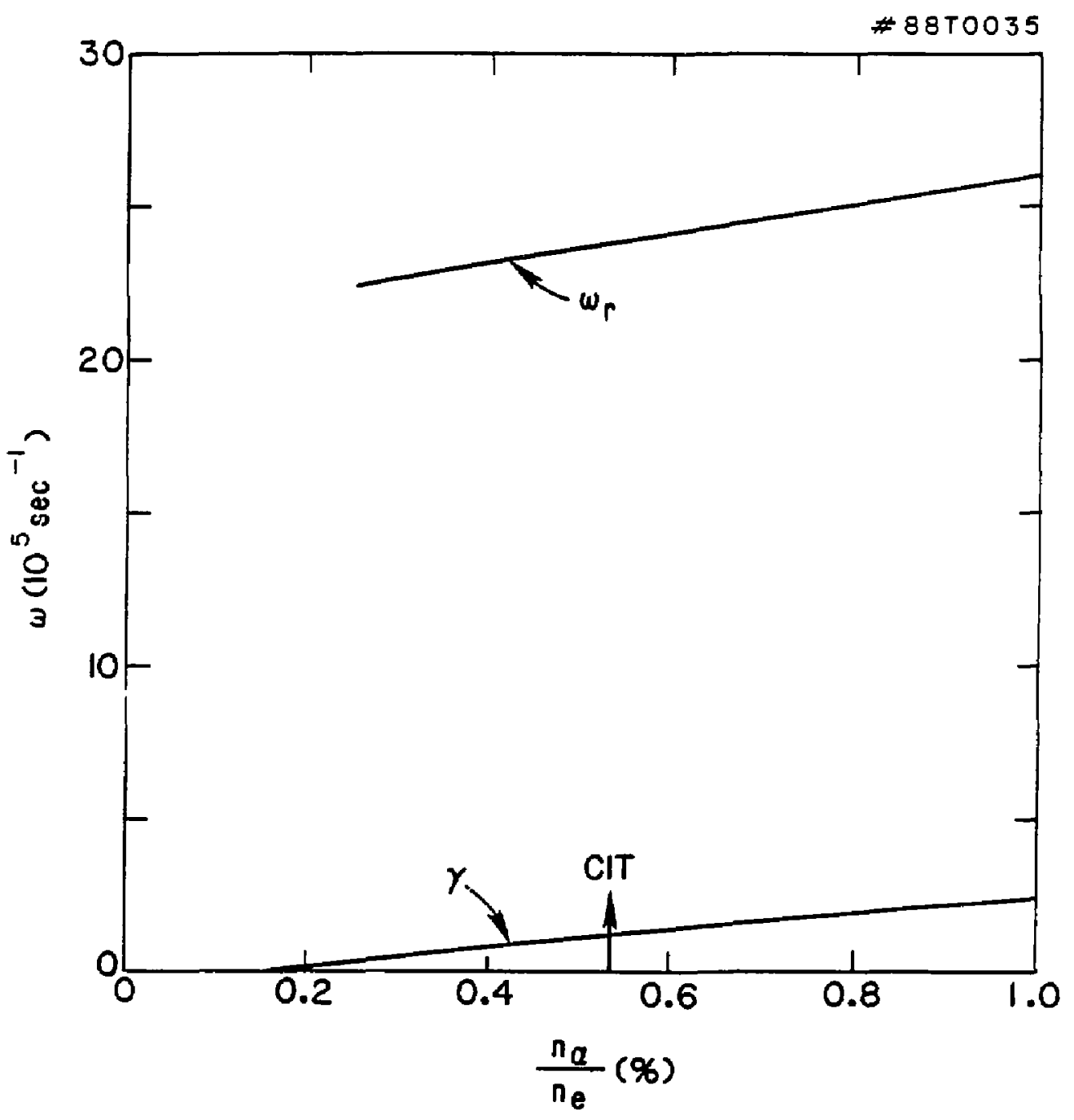

Fig. 8 


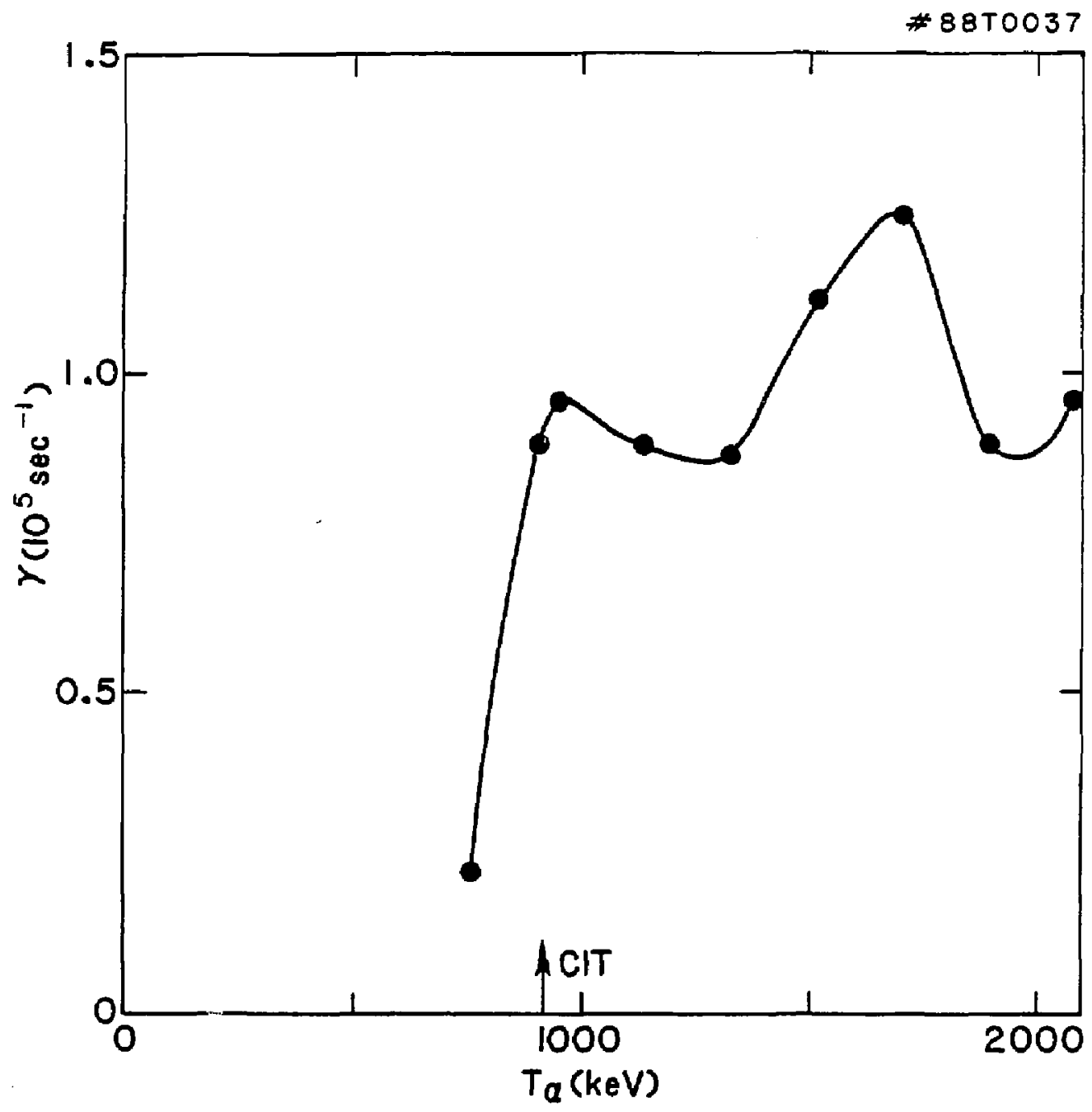

Fig. ? 
\# 88T0044

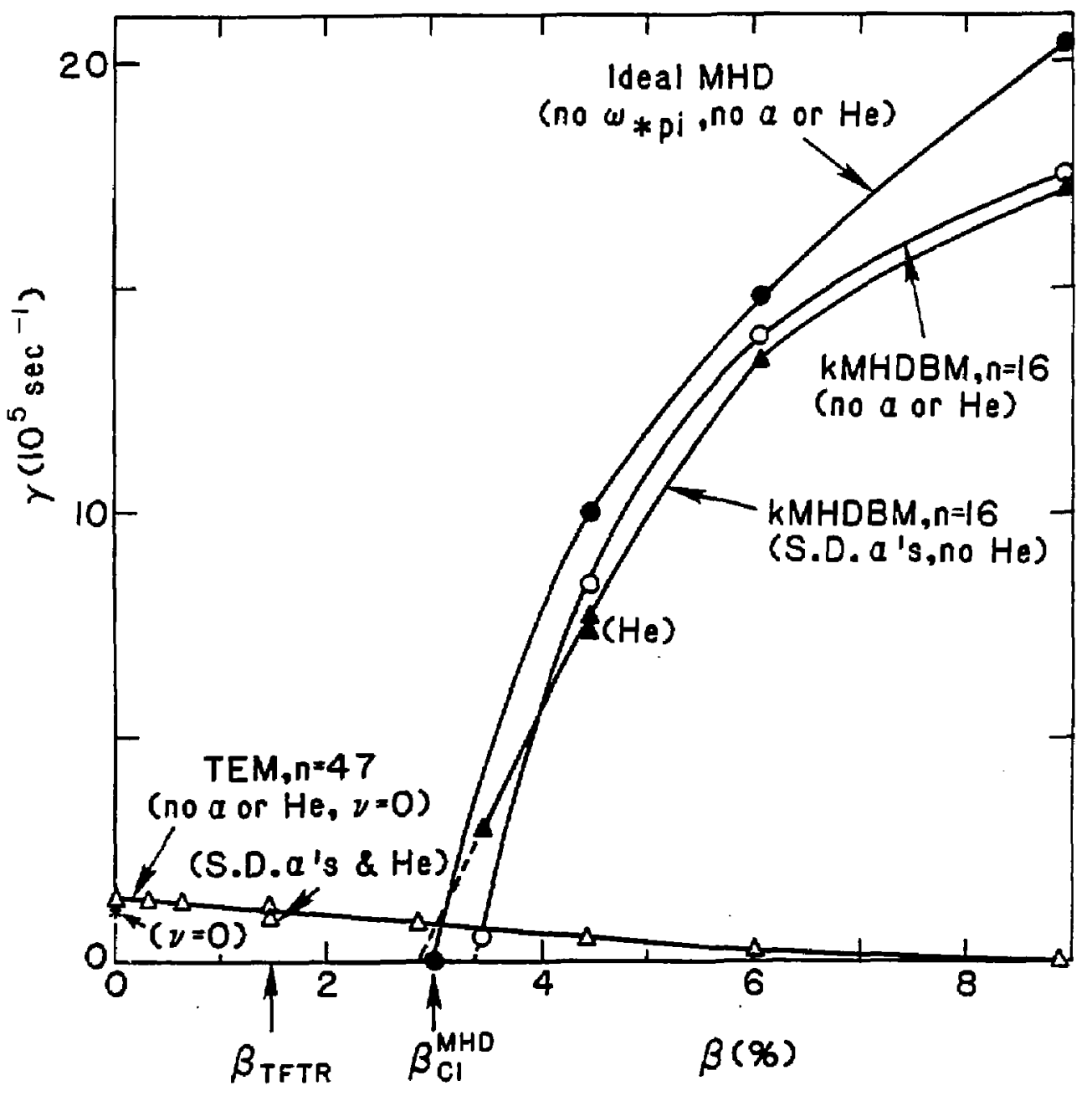

Fig. 10 


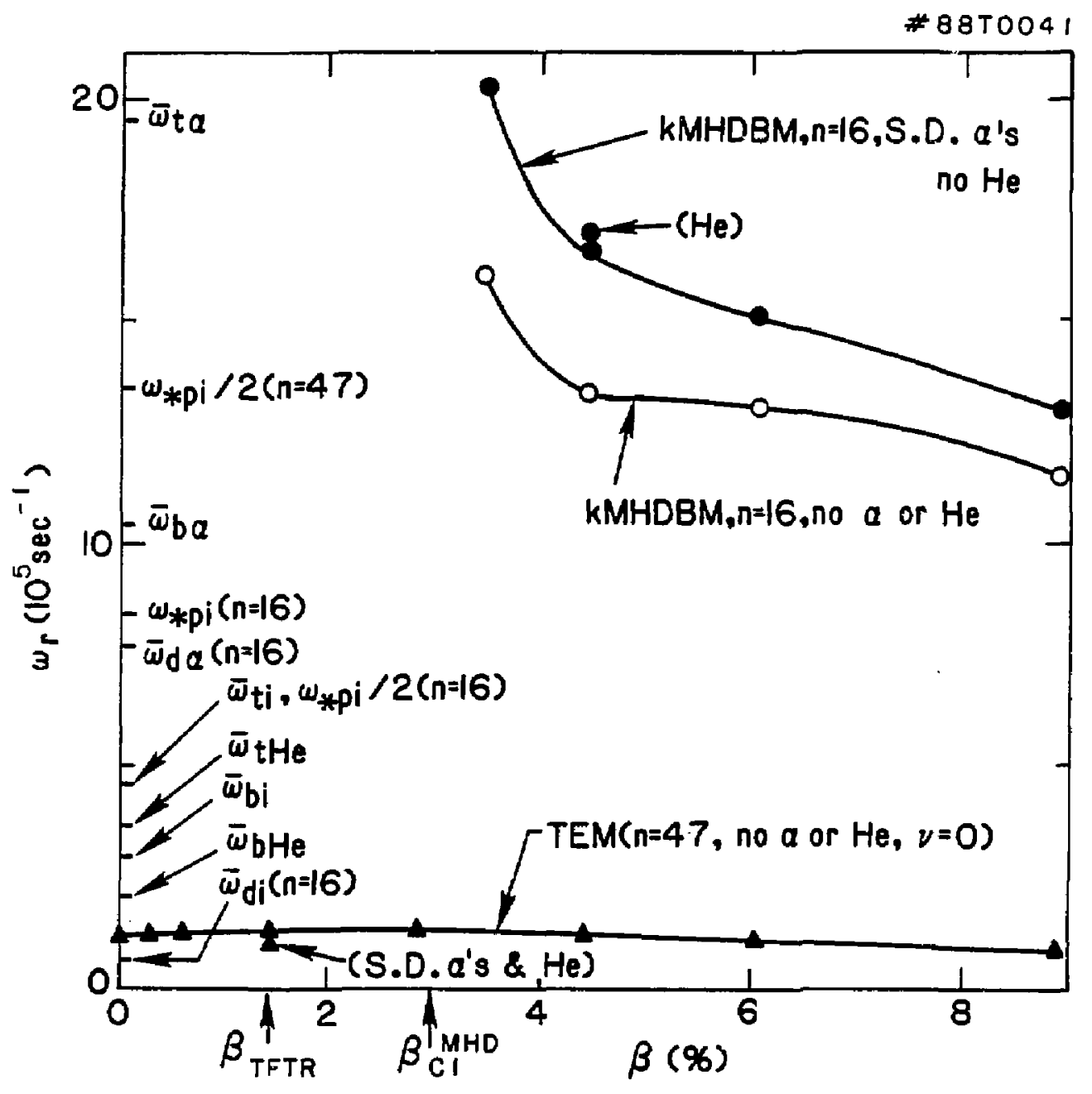

Fig. 11 


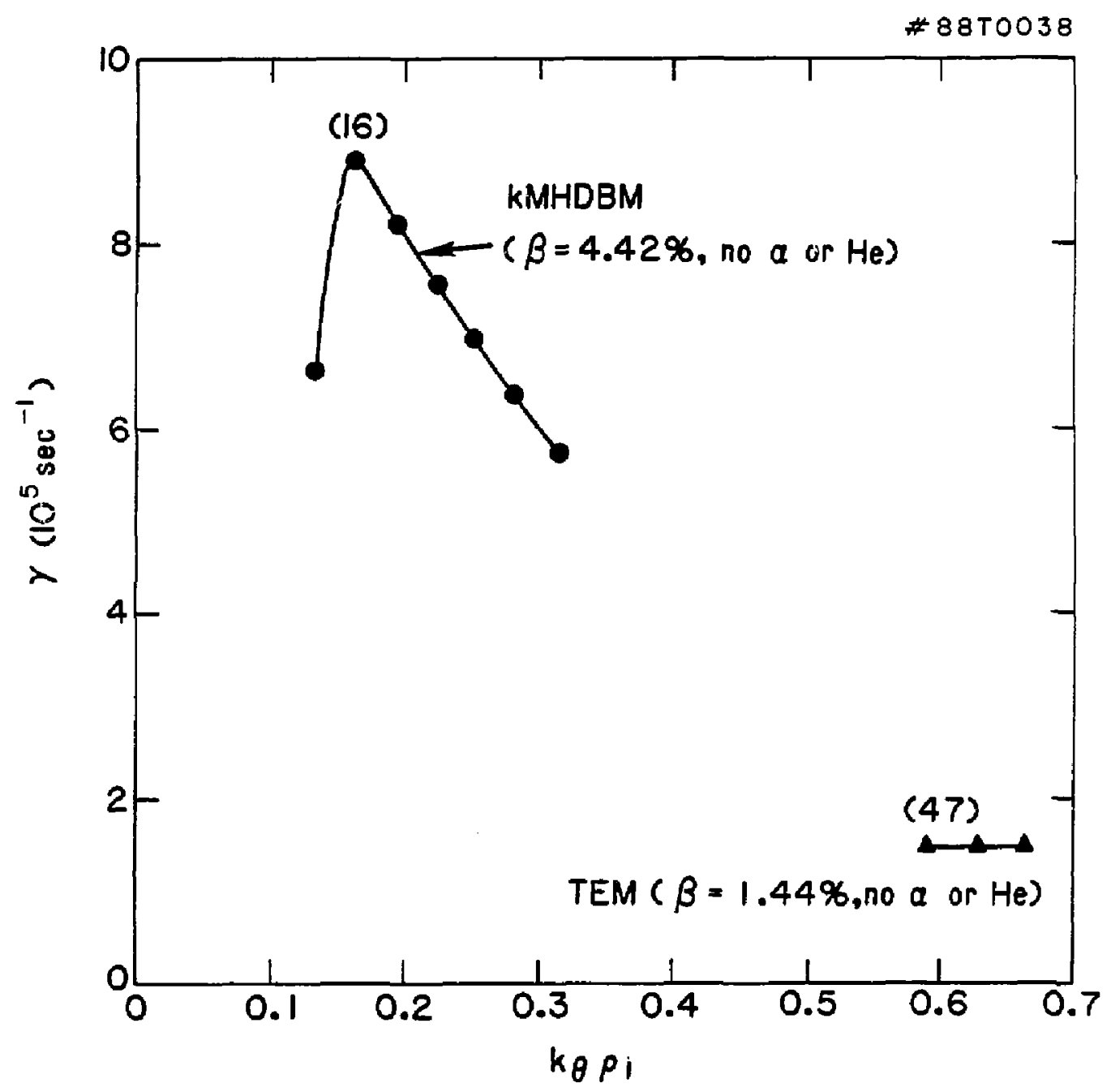

Fig. 12 
Or. Frank J. Pooloni, Univ of Wol iongong, AUSTRALIA Prof, M.H. Erennan, Univ Sydney, AUSTRALIA

Plasma Research Lab., Australian Mat. Univ., AUSTRALIA

Prof. I.R. Jones, FI incers Univ., AUSTRALIA

Prof. F. Cad, inst Theo Phys A AustRIA

Prot. M. Heindler, Institut f ur Theoretische Physlk, AuSTRIA

- M. Goossens, Astronomisch Instituut, BELGILM

Ecole Royale Militaire, Lat de Phys Plasmas, Belgiv

Commission-Eurogean, Dg-XII Fusion Prog, BELGIUM

Prof, R. Boucique, Laboratorium veor Natuurkunde, BELGIUM

Or. P.H. Sakanaka, Instituto Fisica, BRAZIL

instituto De Pesquisas Espacidasi-INPE, BRAzll

Documents Office, Atomic Energy of Canada Limitad, CAMADA

Dr. M.P. Bachynski, MPQ Tochnologies, Inc., CANADA

Dr. H.M. Skarsgard, University of Saskatchaman, CANAOA

Or. H. Barnard, University of British Columbla, CAMADA

Prof. J. Teichmann, Univ. of Hontreal, CAMADA

Prof. S.A. Sreenivasan, University of Calgary, CAMMA

Prof. Tudor W. Johnston. IMRS-Energl\$, CAMADA

Or. C.R. James, Univ. of Alberta, CANADA

Dr. Peter Lukac, Komanskoho Univ, CZECHOSLOVAKIA

The Librarian, Culha Laboratory. ERLLAND

The Librarian, Rutherford Appleton Laboratory, ENcLAND

itrs. S.A. Hutchinson, JET Library, ENGLAND

C. Mouttat, Lab. de Physique des Milieux lorises, fRAnce

J. Radet, CEN/CMOARACHE - Gat 506, FRANCE

- Univ. of Ioannina, Library of Physics Dept. GPEECE

Or. Ton Mual, Acadeay Bibliographic Ser.., hows kows

Preprint Library, Hungarian Academy of Sciences, HUWARY

Dr. 8. Dasgupta, Saha Inst of Nucl. Phys., IMDIA

Dr. P. Xaw, Institute for Plasma Rasearch, INDiA

Or. Philip Rosenau, Israel Inst. Tech, ISRAEL

Librarian, Int'I Ctr Theo Phys, ITALY

Prof, G. Rostagni, Univ Di Padova, ITALY

Miss Clelia De Palo, Assoc EURATOH-ENEA, ITAEY

Giblioteca, Institute di fisica del Plasada, ITALY

Dr. H. Yamato, Toshiba Res \& Dev, JAPNM

Prof. 1. Kawakani, Atomic Energy Res. Institute, JAPAN

Prof, Kyoji Nishikamo, Univ of Hiroshima, JAPAN

Direc. Dept. Large Tokomak Ros. JAERI, JAPAN

Prof. Satosh; Itoh, Kyushu University, JAPAN

Reseerch info Center. Nagoyd University, JAPAH

Prof, 5. Tanaka, Kyoto University, JAPAN

Library, Kyoto University, JAPAN

Prof. Nobuyuki Inowe, University of Tokyo, JAPAN

S. Mor $i$, JAERI, JAPAN

Librarian, Korea Advanced Energy Res. Institute, KOAEA

Prof. 0.1. Choi, Adv. Inst Sci \& Tuch, KOREA

Prof. 0.5. Liloy, University of Maikato, NEN ZEALAMC

Institute of PIAsma Physics, PEOPLE'S REPUBLIC OF CHINA

Librarian, Institute of Phys., PEDPLE'S REPU6LIC OF CHINA

Library, Tsing Hua University, PEOPLE'S REPUBLIC OF CHIMA
2. Li, Southwast Inst. Physics, PEOPLE'S REPUBLIC OF CHINA Prof. J.A.C. Cabral, Inst Suderior Teenico, PORTLGaL Dr. Oetavian Petrus, AL I CUZA University, ROMANIA

Dr. Johan de Villiers, fusion studies, AEC, SO AFRICA Prof. M.A. Hellbarg, University of Natal, SO AFRICA C.I.E.M.A.T., Fusion Div. Library, SPAIN Or. Lennart Stent Ho, University of UAAEA, SHEDEN Llarary, Royal Inst Tech, SWEDEN

Prof. Hans Wilhelmson, Cholmars Univ Tech, SwEDEN

Centre Phys des Plasmas, Ecolo Polytech Fed, SWITZERLAND Gibl lotheak, fom-Inst Voor PI asma-Fysica, THE NETHERLANDS Or. 0.0. Ryutav, Siberian Acad Sei, USSR

Or. G.A. El iseev, Kurchatov Institure, USSR

Dr. V.A. Glukhikh, Inst Electrophysical Apparatus, USSR

Or. V.r. Tolok, Inst. Phys. Tech. USSR

Or. L.H. Kovrizhnykh, Institute Gen. Physics, USSR

Nuclear Res. Establishment, Julich Ltd., W. GERMANY

Bibliothek, Inst. Fur Plasmatorschung, W. GERMANY

Dr. K. Schindler, Ruhr Universitat Bochum. W. GERMANY

ASOEX Rouding Pon, IPP Nax-PI anck-l nstitut fur

Pla mysik, W. GePanany

Librarian, Max-Planek Institut, W. GERMANY

Prof. R.K. Jonev, Inst Phys, yugoslavia 